OPEN ACCESS

Edited by:

David Golomb,

Ben-Gurion University of the Negev,

Israel

Reviewed by:

Alla Borisyuk,

University of Utah, USA

Robert H. Lee,

Emory University, USA

*Correspondence:

Gemma Huguet

gemma.huguet@upc.edu

${ }^{\dagger}$ These authors have contributed equally to this work.

Received: 29 July 2016 Accepted: 16 January 2017 Published: 02 February 2017

Citation:

Huguet G, Meng X and Rinzel J (2017)

Phasic Firing and Coincidence Detection by Subthreshold Negative Feedback: Divisive or Subtractive or, Better, Both

Front. Comput. Neurosci. 11:3. doi: 10.3389/fncom.2017.00003

\section{Phasic Firing and Coincidence Detection by Subthreshold Negative Feedback: Divisive or Subtractive or, Better, Both}

\author{
Gemma Huguet ${ }^{1 * t}$, Xiangying Meng ${ }^{2 t}$ and John Rinzel ${ }^{3,4}$ \\ ${ }^{1}$ Departament de Matemàtiques, Universitat Politècnica de Catalunya, Barcelona, Spain, ${ }^{2}$ Biology Department, University of \\ Maryland, College Park, MD, USA, ${ }^{3}$ Center for Neural Science, New York University, New York, NY, USA, ${ }^{4}$ Courant Institute \\ of Mathematical Sciences, New York University, New York, NY, USA
}

Phasic neurons typically fire only for a fast-rising input, say at the onset of a step current, but not for steady or slow inputs, a property associated with type III excitability. Phasic neurons can show extraordinary temporal precision for phase locking and coincidence detection. Exemplars are found in the auditory brain stem where precise timing is used in sound localization. Phasicness at the cellular level arises from a dynamic, voltage-gated, negative feedback that can be recruited subthreshold, preventing the neuron from reaching spike threshold if the voltage does not rise fast enough. We consider two mechanisms for phasicness: a low threshold potassium current (subtractive mechanism) and a sodium current with subthreshold inactivation (divisive mechanism). We develop and analyze three reduced models with either divisive or subtractive mechanisms or both to gain insight into the dynamical mechanisms for the potentially high temporal precision of type III-excitable neurons. We compare their firing properties and performance for a range of stimuli. The models have characteristic non-monotonic input-output relations, firing rate vs. input intensity, for either stochastic current injection or Poisson-timed excitatory synaptic conductance trains. We assess performance according to precision of phase-locking and coincidence detection by the models' responses to repetitive packets of unitary excitatory synaptic inputs with more or less temporal coherence. We find that each mechanism contributes features but best performance is attained if both are present. The subtractive mechanism confers extraordinary precision for phase locking and coincidence detection but only within a restricted parameter range when the divisive mechanism of sodium inactivation is inoperative. The divisive mechanism guarantees robustness of phasic properties, without compromising excitability, although with somewhat less precision. Finally, we demonstrate that brief transient inhibition if properly timed can enhance the reliability of firing.

Keywords: phasic firing, type III excitability, divisive, subtractive, coincidence detection, phase-locking 


\section{INTRODUCTION}

Phasic neurons may fire at the onset of a step input, typically once, but not during the steady portion and not for slowly varying inputs. This property of phasic firing is often called type III excitability, in contrast to repetitive firing for slow inputs for type I and II excitable neurons (Hodgkin, 1948; Svirskis et al., 2002; Izhikevich, 2007; Prescott et al., 2008a; Meng et al., 2012; Rinzel and Huguet, 2013). Phasic neurons can show extraordinary temporal precision for phase locking and coincidence detection. Some examples of phasic neurons include: auditory brain stem neurons that are involved with precise timing computations (Oertel, 1999; Schnupp and Carr, 2009; Carr and Macleod, 2010), some spinal cord neurons (Prescott and De Koninck, 2002; Prescott et al., 2008a), and even the squid giant axon (Clay et al., 2008).

Underlying phasicness is a dynamic, voltage-gated, negative feedback that can be recruited subthreshold, preventing the neuron from reaching spike threshold if an increasing input does not rise fast enough. Various cellular mechanisms can implement the dynamic negative feedback. Type III excitability may be due to an outward current (say, potassium $\mathrm{K}^{+}$) that activates relatively fast for subthreshold voltages (Rathouz and Trussell, 1998; Svirskis et al., 2002; Rothman and Manis, 2003; Prescott et al., 2008a). Thus, if an input current depolarizes a cell too slowly, the outward $\mathrm{K}^{+}$current can activate and oppose the voltage rise, limiting the depolarization to subthreshold levels. In contrast, with rapid depolarization due to a fast-rising input current, the $\mathrm{K}^{+}$current lags, allowing subsequent spike generation. During the spike, the $\mathrm{K}^{+}$current strongly activates, precluding subsequent spikes, and therefore, repetitive firing.

This subtractive ( $\mathrm{K}^{+}$current) mechanism is not the only way to generate type III excitability. Indeed, a divisive mechanism, such as a fast-activating, but transient, inward (sodium $\mathrm{Na}^{+}$ or calcium) current with suitably recruitable inactivation, can also generate type III excitability (Prescott and De Koninck, 2002; Svirskis et al., 2004; Gai et al., 2009; Platkiewicz and Brette, 2011). The conceptual framework is the same. If the cell depolarizes slowly, the inactivation process develops before the inward current can activate and the cell will not fire a spike. If the depolarization is fast enough before inactivation of the inward current can occur, a spike (and only one spike) is produced.

In some auditory neurons both subtractive and divisive mechanisms contribute to phasic firing (Svirskis et al., 2004; Scott et al., 2010); it appears that inactivation of sodium current $I_{\mathrm{Na}}$ is $V$-gated at unusually low $V$ values. Neurons in the auditory brain stem, where precise timing is important for sound localization (Oertel, 1983; Reyes et al., 1994; Oertel, 1999; Rothman and Manis, 2003), show extraordinary temporal precision (on subms time scales) for phase locking and coincidence detection. One may wonder what is the contribution of each mechanism in shaping the properties of the system and what is gained by having two feedback processes.

To address these questions we utilize reduced 2 and 3-variable versions (Meng et al., 2012) of an 8-variable biophysicallybased model developed by (Rothman and Manis, 2003). This 8 -variable model, that we refer to as RM03, has been widely used for modeling the phasic firing of cells in the auditory brainstem. In RM03, the dominant mechanism for phasicness is a low-threshold potassium current $\left(I_{\mathrm{KLT}}\right)$ (subtractive dominant mechanism). Phasic behavior is lost if the conductance of $I_{\mathrm{KLT}}$ is frozen at its resting value, but phasic firing can be restored with a divisive negative feedback mechanism by left-shifting the $V$-dependence of steady-state inactivation gating for sodium current. We characterize the features of the subtractive and divisive mechanisms by isolating each in our reduced models; one has only subtractive ( $\mathrm{S}$ model), one only divisive (D model), and a third has a combination of both (C model).

Our reduced models permit mathematical analysis, including phase plane analysis, and thereby prediction and insight into the phasic firing properties. Using tools from bifurcation theory we can assess the range of conductances for which the neuron models show type III excitability. We observe that the subtractive mechanism if considered alone (i.e., if the $I_{\mathrm{Na}}$ is non-inactivating) requires a strong reduction of $\mathrm{Na}^{+}$conductance in order to preserve its phasic properties and prevent a highly depolarized state for strong inputs (depolarized state of "lockup"). The presence of a divisive mechanism combined with a subtractive one, guarantees the robustness of the phasic properties of the system with respect to changes in channel density.

In this comparative study, we use a range of different stimuli that help us to elucidate the different contributions of each mechanism to the output properties. As a function of a steady input's amplitude the three models show no bifurcation to repetitive activity. Indeed, there is firing only in the presence of input fluctuations and firing probability shows strong sensitivity to input variance (Higgs et al., 2006; Lundstrom et al., 2008, 2009; Gai et al., 2009). In order to quantify this sensitivity, we compared the models' responses to ramps and periodic inputs, as well as their input-output curves (firing rate vs. mean input and varying noise strength, and firing rate vs. excitatory input frequency with random maximal conductance). We observe that the low-threshold $\mathrm{K}^{+}$conductance in the $\mathrm{S}$ model prevents excessive depolarization, thus keeping the cell in the proper voltage operating range, and provides robustness to small input fluctuations. On the contrary, the D model has higher input resistance, thus more vulnerable to input fluctuations and mean depolarization, which ultimately leads to excitability loss from $I_{\mathrm{Na}}$ inactivation.

In order to assess coincidence-detection and phase-locking, we inject periodic trains of excitatory inputs, created from many small excitatory postsynaptic conductances (EPSGs) with random event times, that can be more or less synchronized. We vary the input frequency and vector strength (a measure of coincidence), and we compute the output firing rate (a measure of entrainment) and vector strength (a measure of phase locking). We find that while both mechanims show enhancement of vector strength, subtractive spikers can out-perform divisive spikers for temporal precision and coincidence detection, especially in the presence of a background noise. Finally, we explore the effects of background constant inhibition as well as timed inhibition and we observe that inhibition can have contrasting effects on the firing rate, positive or negative, but the $\mathrm{D}$ model shows less sensitivity to the arrival time of the inhibitory 
inputs compared to the $\mathrm{S}$ and $\mathrm{C}$ models, especially at high frequencies.

Our results suggest that phasic neurons equipped with two negative feedback processes are more robust to changes in applied currents and conductance densities than models that possess only one negative feedback mechanism, while they show stronger coincidence detection properties.

\section{METHODS}

\subsection{Neuron Models}

Rothman and Manis (2003) developed a Hodgkin-Huxley-like neuron model that has been widely used for modeling the phasic firing of cells in the auditory brainstem. It consists of a sodium current $I_{\mathrm{Na}}$, a high-threshold $\left(I_{\mathrm{KHT}}\right)$ and a low-threshold $\left(I_{\mathrm{KLT}}\right)$ potassium currents, a hyperpolarization-activated cation current $I_{h}$ and a leak current $I_{\mathrm{lk}}$. The current balance equation has the following expression:

$$
C \frac{d V}{d t}=-I_{\mathrm{Na}}-I_{\mathrm{KHT}}-I_{\mathrm{KLT}}-I_{\mathrm{h}}-I_{\mathrm{lk}}+I(t),
$$

where $C$ is the membrane capacitance, $V$ is the membrane voltage and $I(t)$ is the external input current. Each ionic current $I_{i}$ is governed by an activation and/or inactivation variable, and Equation (1) can be written as

$$
\begin{aligned}
C \frac{d V}{d t}= & 2\left[-\bar{g}_{\mathrm{Na}} m^{3} h\left(V-E_{\mathrm{Na}}\right)-\bar{g}_{\mathrm{KHT}}\left(0.85 n^{2}+0.15 p\right)\left(V-E_{\mathrm{KHT}}\right)\right. \\
& \left.-\bar{g}_{\mathrm{KLT}} w^{4} z\left(V-E_{\mathrm{K}}\right)-\bar{g}_{h} r\left(V-E_{h}\right)-\bar{g}_{\mathrm{lk}}\left(V-E_{\mathrm{lk}}\right)\right]+I(t),
\end{aligned}
$$

where $\bar{g}_{i}$ and $E_{i}$ are respectively the maximal conductances and reversal potentials for the ionic current $i=\mathrm{Na}, \mathrm{KHT}, \mathrm{KLT}, \mathrm{h}, \mathrm{lk}$. Maximal conductances and channel gating rates are multiplied by a factor of 2 and 3, respectively, as in Gai et al. (2009), to mimic the brain slices during whole cell recordings at temperature $32^{\circ} \mathrm{C}$.

Of particular interest for our problem is the dynamics of activation of low-threshold potassium current $I_{\text {KLT }}$ governed by the variable $w$ and the dynamics of inactivation of the transient sodium current $I_{\mathrm{Na}}$ governed by the variable $h$.

To highlight the primary biophysical mechanisms for phasic firing properties and to facilitate our analysis, we proceed as in Meng et al. (2012), and develop 3 reduced versions of the 8-variable RM03 model by identifying and approximating some nonessential features for the model's excitability and spike generation mechanism. Namely, we set $m=m_{\infty}(V)$, we freeze the inactivation gating variable $z$ of $I_{\text {KLT }}$ and the activation gating variable $r$ of $I_{h}$, and we remove $I_{\mathrm{KHT}}$ (see Meng et al., 2012 for more details and a justification of this simplification).

The reduced models considered are two 2-variable models, both divisive and subtractive dominant versions, and a third 3-dimensional model that combines both mechanisms.

\subsubsection{Subtractive (S) Model}

In order to isolate the subtractive mechanism, we disable the subthreshold dynamic negative feedback provided by inactivation of $I_{\mathrm{Na}}$ by freezing the inactivation variable $h$ at its value at the resting state for the other two models. To do so and still retain the type III excitability property, we need to reduce $\bar{g}_{\mathrm{Na}}$ (see Appendix B in Supplementary Material). The model then writes as:

$$
\begin{aligned}
C \frac{d V}{d t}= & -2\left(\bar{g}_{\mathrm{Na}} m_{\infty}(V)^{3} h_{0}\left(V-E_{\mathrm{Na}}\right)+\bar{g}_{\mathrm{KLT}} w^{4} z_{0}\left(V-E_{\mathrm{K}}\right)\right. \\
& \left.+g_{1}\left(V-E_{1}\right)\right)+I \\
\frac{d w}{d t}= & 3 \frac{w_{\infty}(V)-w}{\tau_{w}(V)}
\end{aligned}
$$

where $C=12 \mathrm{pF}, \bar{g}_{\mathrm{Na}}=177 \mathrm{nS}, h_{0}=0.22, \bar{g}_{\mathrm{KLT}}=200 \mathrm{nS}$, $z_{0}=0.662, g_{l}=4.97 \mathrm{nS}, E_{N a}=55 \mathrm{mV}, E_{K}=-70 \mathrm{mV}$ and $E_{l}=-52.024 \mathrm{mV}$. Notice that we obtained $g_{1}$ and $E_{1}$ from the original RM03 model by setting $g_{1}=\bar{g}_{\mathrm{h}} r_{0}+\bar{g}_{1 \mathrm{k}}$ and $E_{1}=$ $1 / g_{1}\left(\bar{g}_{\mathrm{h}} r_{0} E_{\mathrm{h}}+\bar{g}_{\mathrm{lk}} E_{\mathrm{lk}}\right)$.

The steady-state functions $m_{\infty}(V)$ and $w_{\infty}(V)$ (see Figure 1A) are given by

$$
m_{\infty}(V)=\left(1+e^{-(V+38) / 7}\right)^{-1},
$$

and

$$
w_{\infty}(V)=\left(1+e^{(-(V+48) / 6}\right)^{-1 / 4},
$$

respectively, and the time function for $w$ (see Figure 1B) is

$$
\tau_{w}(V)=1.5+\frac{100}{6 e^{(V+60) / 6}+16 e^{-(V+60) / 45}} .
$$

\subsubsection{Divisive (D) Model}

We can disable the subthreshold dynamic negative feedback provided by activation of $I_{\mathrm{KLT}}$ by freezing the conductance $g_{\mathrm{KLT}}$ (activation and inactivation variables $w$ and $z$ ) to its value at the resting state. In this case, the only subthrehsold dynamic negative feedback that is left in the model is sodium inactivation, but in order to ensure phasic firing for a steady input, we manipulated the model so that sodium inactivation is shifted to lower values of voltage (i.e., $\left.h_{\infty}(V+6)\right)$. The model then reduces to:

$$
\begin{aligned}
C \frac{d V}{d t}= & -2\left(\bar{g}_{\mathrm{Na}} m_{\infty}(V)^{3} h\left(V-E_{\mathrm{Na}}\right)+\bar{g}_{\mathrm{KLT}} w_{0}^{4} z_{0}\left(V-E_{\mathrm{K}}\right)\right. \\
& \left.+g_{1}\left(V-E_{1}\right)\right)+I \\
\frac{d h}{d t}= & 3 \frac{h_{\infty}(V)-h}{\tau_{h}(V)}
\end{aligned}
$$

where $w_{0}=0.512, \bar{g}_{\mathrm{Na}}=500 \mathrm{nS}$ and the other parameter values are as in the $\mathrm{S}$ model.

The steady-state function $h_{\infty}(V)$ (see Figure 1A) is given by

$$
h_{\infty}(V)=\left(1+e^{\left(V+h_{\text {shift }}+65\right) / 6}\right)^{-1},
$$

and the time function for $h$ (see Figure 1B) is

$$
\tau_{h}(V)=\frac{100}{7 e^{\left(V+60+h_{\text {shift }}\right) / 11}+10 e^{-\left(V+60+h_{\text {shift }}\right) / 15}}+0.6,
$$

with $h_{\text {shift }}=6$. 

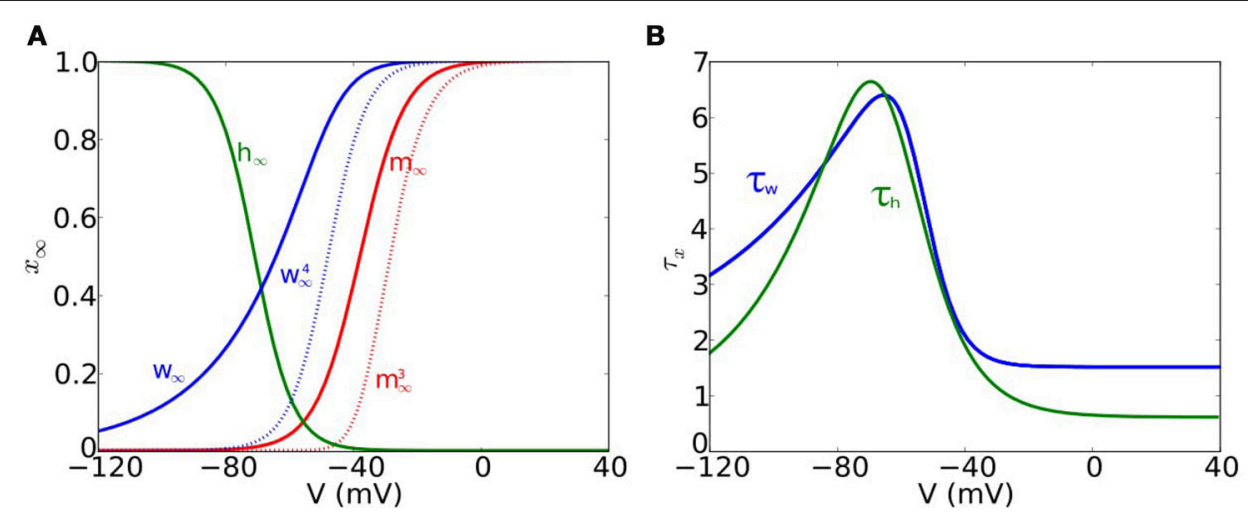

FIGURE 1 | Voltage-dependent functions of the models. (A) Voltage-dependent steady-state functions for the gating variables of the ionic currents: sodium activation $m_{\infty}$ (solid red), sodium inactivation $h_{\infty}$ (green) and potassium activation $w_{\infty}$ (solid blue). We also include $w_{\infty}^{4}$ (dotted blue) and $m_{\infty}^{3}$ (dotted red). (B) Time constant functions for sodium inactivation $\tau_{h}$ (green) and potassium activation $\tau_{W}$ (blue).

\subsubsection{Combined (C) Model}

The model has both dynamic subthreshold negative feedback mechanisms: activation of $I_{\mathrm{KLT}}$ (as modeled for the $\mathrm{S}$ model) and inactivation of $I_{\mathrm{Na}}$ (as modeled for the $\mathrm{D}$ model). The voltage equation is given by:

$$
\begin{aligned}
C \frac{d V}{d t}= & -2\left(\bar{g}_{\mathrm{Na}} m_{\infty}(V)^{3} h\left(V-E_{\mathrm{Na}}\right)+\bar{g}_{\mathrm{KLT}} w^{4} z_{0}\left(V-E_{\mathrm{K}}\right)\right. \\
& \left.+g_{1}\left(V-E_{1}\right)\right)+I \\
\frac{d w}{d t}= & 3 \frac{w_{\infty}(V)-w}{\tau_{w}(V)} \\
\frac{d h}{d t}= & 3 \frac{h_{\infty}(V)-h}{\tau_{h}(V)}
\end{aligned}
$$

where $\bar{g}_{\mathrm{Na}}=500$ and the other parameter values are as before. The functions $h_{\infty}(V)$ and $\tau_{h}$ are given in Equations (7) and (8), respectively (as in the $\mathrm{D}$ model), and $w_{\infty}$ and $\tau_{w}$ are given in Equations (4) and (5), respectively (as in the S model).

\subsection{Time Scales Ratio}

We compute the ratio between voltage and gating variable time constants for the divisive and the subtractive models.

We define the total conductance as

$$
g_{\text {tot }}(V, h, w)=2\left(g_{\mathrm{Na}}(V, h)+g_{\mathrm{KLT}}(V, w)+g_{1}\right) .
$$

The ratio for the divisive model is given by:

$$
r(V, h)=\frac{3 C}{g_{\text {tot }}\left(V, h, w_{0}\right) \tau_{h}(V)},
$$

and for the subtractive:

$$
r(V, w)=\frac{3 C}{g_{\text {tot }}\left(V, h_{0}, w\right) \tau_{w}(V)} .
$$

Notice that values of $r$ close to 1 indicate that the two variables evolve on a similar time scale, while values of $r$ close to 0 indicate that the voltage evolves on a much faster time-scale than the gating variable.

\subsection{Inputs}

We consider several types of deterministic inputs: steps, ramps and also a half-wave rectified sinusoidal input $I(t)$ with amplitude $A$ and frequency $\omega$,

$$
I(t)=A[\sin (2 \pi \omega t)]^{+},
$$

where $[\cdot]^{+}=\max (\cdot, 0)$. In some cases we inject external noisy input of the form $I_{\text {noise }}=C \sigma \eta(t)$, where $\eta(t)$ is a "white noise" process.

We also consider conductance based synaptic-like currents. Each synaptic site generates a minimal excitatory or inhibitory postsynaptic conductance (EPSG/IPSG) that is modeled as an alpha function with time constant $\tau_{\text {syn }}=0.3 \mathrm{~ms}$, unless otherwise stated, and maximal conductance $g_{\text {max }}$ :

$$
g_{s y n}(t)=g_{\max } \frac{t}{\tau_{s y n}} e^{1-t / \tau_{s y n}} .
$$

Thus, conductance-based synaptic currents have the form:

$$
I_{\text {syn }}(t)=g_{\text {syn }}\left(t-t_{s}\right)\left(V-E_{\text {syn }}\right),
$$

for $t \geq t_{s}$, where $E_{\text {syn }}=0$ and $E_{\text {syn }}=-75 \mathrm{mV}$ for excitatory and inhibitory synaptic inputs, respectively, and $t_{s}$ is the pre-synaptic spike time.

For the case of steady (but random) synaptic inputs, the synaptic event times are Poisson distributed with a given frequency and their maximal conductances are modeled as a summation of $N=7$ independent miniature EPSGs with fixed amplitude, each of which occurs with probability $p=1 / 2$. Thus, maximal conductances for composite EPSGs are discrete and modeled with a binomial distribution, $B(N, p)$, where $N=7$ is the maximum number of $\mathrm{mEPSG}$ each with probability $p=0.5$ of occurence. Thus, the mean number of mEPGS is 3.5, i.e., there will be $50 \%$ probability for $N \leq 3$ and $50 \%$ probability for $N \geq 4$.

In order to test coincidence detection, we inject periodically modulated excitatory synaptic inputs from several fibers/sites as in Jercog et al. (2010), similar to what might occur in vivo 
(Joris et al., 1994). Each cycle's composite input was generated from eight small (mini) excitatory postsynaptic conductances (mEPSGs) modeled as alpha functions with fixed amplitude and event times per site drawn from a von Mises distribution independently at each cycle. The von Mises probability density function for the angle $\theta$ is given by:

$$
f(\theta, a, b)=\frac{e^{b \cos (\theta-a)}}{2 \pi I_{0}(b)},
$$

where $a=1 / 4$ is the mean and $b$ is the temporal coherence ( $b=0$ corresponds to a uniform distribution and as $b$ increases the distribution becomes more concentrated about the angle $a$ ), and $I_{0}(x)$ is the modified Bessel function of order 0 . Notice that to vary $b$ is equivalent to vary the vector strength of the periodic input train. The vector strength (VS), also known as "synchronization index" (Goldberg and Brown, 1969), is a measure of how clustered are events over a cycle. To compute the VS one associates to each event time a vector on the unit circle with a phase angle and computes the mean vector. The VS is given by the length of the mean vector. Perfect clustering is obtained when $V S=1$. The relationship between VS and the temporal coherence $b$ of the input distribution is given by:

$$
V S(b)=I_{1}(b) / I_{0}(b),
$$

where $I_{0}(x)$ and $I_{1}(x)$ are the modified Bessel function of order 0 and 1 , respectively.

In our simulations we chose maximal conductances for individual mEPSGs according to the following criteria: for low input strength, we pick $g_{\text {max,e }}$ so that six coincident inputs, but not less, generate a composite EPSG that exceeds threshold for spike at rest, and for high input strength we reduce this number to four coincident inputs. Notice, that since the EPSG threshold for spike is different for each model, we used different values of $g_{\text {max }, e}$ for each model.

In order to test the role of timed inhibition we also inject periodic inhibitory inputs generated in the same way as the excitatory ones: composite IPSGs are obtained from mini-IPSGs (mIPSGs) modeled as alpha functions (see Equation 11) with maximal conductance $g_{\text {max }, i}$ and the timings in the arrival of mIPSGs are also drawn from a von Mises distribution. The frequency of the inhibitory input will be the same as for the excitatory input, but we will vary other parameters for inhibition: the temporal coherence $b_{i}$ in the von Mises distribution (Equation 12), the maximal conductance $g_{\text {max }, i}$ and time scale $\tau_{i}$ of the mIPSGs (Equation 11). Since we want explore the role that arrival time of inhibition plays in the maximal response of the system, we also vary the phase difference $\varphi$ between the inhibitory and the excitatory inputs $\left(\varphi=a_{i}-a_{e}\right.$ ( $\bmod 1), \varphi \in[0,1])$, where $a_{i}$ and $a_{e}$ are the means of the von Mises distribution for the inhibitory and the excitatory input trains, respectively. Notice that when $\varphi$ is close to 0 , inhibition just follows excitation, while when $\varphi$ is close to 1 , inhibition just precedes excitation.

\subsection{Criterion for Spike Identification or Detection}

RM03-like models have low input resistance. The high conductance shunts EPSCs as well as spike currents. Strong EPSGs are required to elicit a spike and distinguishing spikes from EPSPs requires care. Although spikes may vary in amplitude, we observe that there is a sharp rise in voltage response when the input exceeds a certain value (see Figure 2C), showing the presence of a regenerative process. Thus, our criterion for spike detection requires that $V$ passes a set level $(-20 \mathrm{mV})$ and that the net intrinsic current when $V=-20 \mathrm{mV}$ is negative, i.e., dominated by sodium current (in order to detect the regenerative process).

\subsection{Simulation Methods}

Equations were integrated numerically in C using an implicit 4th order Runge-Kutta method. Stochastic differential equations had a white noise term and were integrated using an Euler-Maruyama method for stochastic differential equations (Higham, 2001) with time step $\Delta t=0.005 \mathrm{~ms}$, along with a random number generator from the GSL-GNU Scientific Library. The bifurcation diagrams were computed using the Auto feature in XPPAUT. We used Matlab and Python to analyze and plot the data.

\section{RESULTS}

\subsection{Reduced Models with Divisive or Subtractive Mechanisms for Type III Excitability}

We have developed reduced models (with 2 or 3 variables) that show type III excitability (each model fires once only at the onset of a step current and not repetitively thereafter) using two different biological mechanisms, subtractive and divisive, that we managed to isolate.

Our starting point model is a reduced version of the Rothman and Manis model (RM03) (Rothman and Manis, 2003) for phasic neurons in the auditory brainstem (see Equation 1 in Methods), that we previously showed behaves semi-quantitatively and qualitatively as the full model (Meng et al., 2012). This reduced version contains a potassium current that is partially activated at $V_{\text {rest }}$ and can significantly activate for subthreshold voltages, and a transient sodium current that can significantly inactivate for subthreshold voltages. Noting that "threshold" does not correspond to a fixed voltage value for spike initiation, especially in the case of phasic models (Azouz and Gray, 2000; Platkiewicz and Brette, 2010, 2011), we use as threshold the voltage value at which sodium activation (responsible of spike generation) rises sharply (i.e., $m_{\infty}^{3}(V)$ ), which is approximately $-40 \mathrm{mV}$. This voltage value would correspond to spike initiation if the dynamic subthreshold negative feedback factors were not present (see Section Methods for our precise criterion for spike identification). For this reduced RM03 model we also remove the $I_{\mathrm{KHT}}$ current and freeze the activation variable of $I_{h}$, as well as the $I_{\mathrm{KLT}}$ inactivation variable at their resting values $\left(V_{\text {rest }}=\right.$ $-63.6 \mathrm{mV})$. 
A

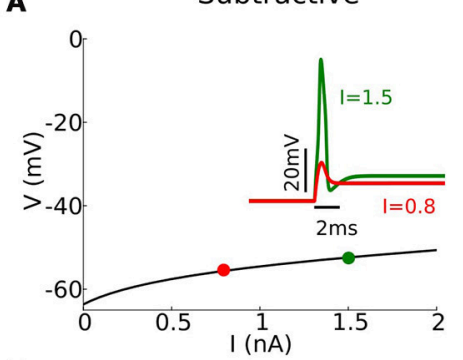

B

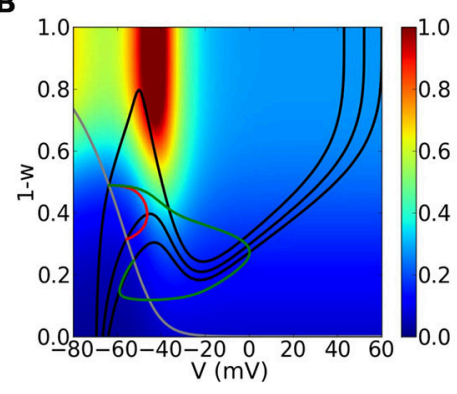

Divisive
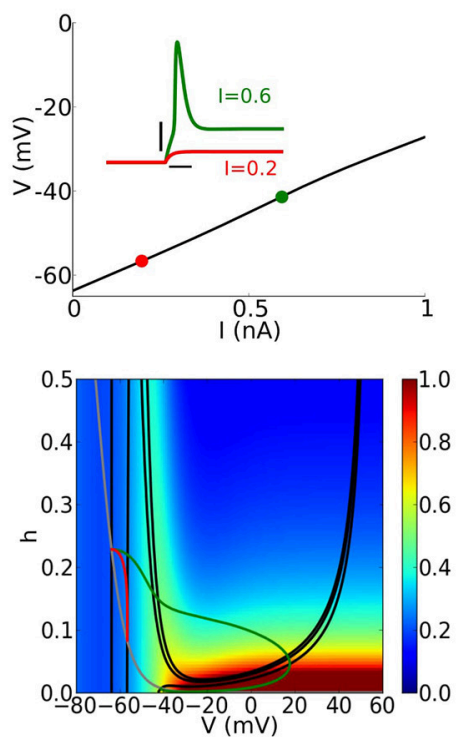

Combined

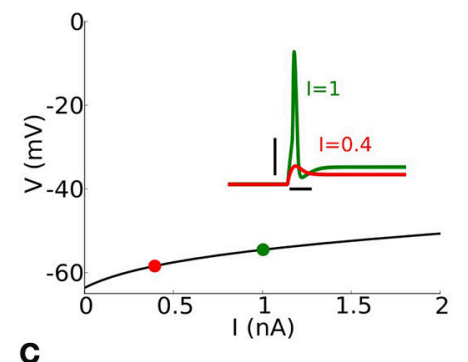

C

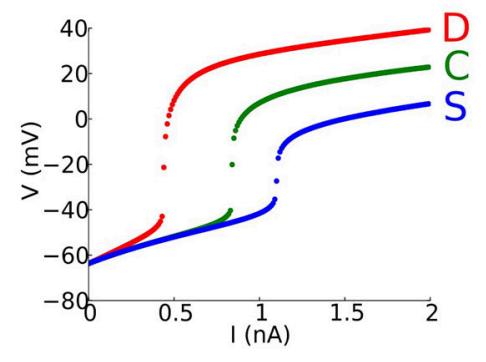

FIGURE 2 | Basic properties of phasic firing. (A) Steady state (fixed point) continuation for S (left), D (center), and C (right) models for a steady current I. Stable fixed point (black solid) does not destabilize. (Inset) Voltage responses to a step current of different amplitudes indicated in each panel. (B) Phase-plane portraits and trajectories corresponding to the same step current as in (A) for $\mathrm{S}$ and D models. (Left, $\mathrm{S}$ model) $V$-nullclines for $I=0,0.8,1.5 \mathrm{nA}$ (solid black curve) and $w$-nullcline (gray curve). Colors indicate ratio between $V$ and $w$ time-scales considering only ratios smaller than 1. (Middle, $\mathrm{D}$ model) $V$-nullclines for $I=0,0.2,0.6 \mathrm{nA}$ (solid black curve) and $h$-nullcline (gray curve). Colors indicate ratio between $V$ and $h$ time-scales considering only ratios smaller than 1. (C) Maximal value of $V$ in response to a step current of varying amplitude ( $x$-axis) for $S$ (blue), D (red), and C (green) models.

Now, we formulate 2-variable models that isolate the two negative feedback mechanisms. The Substractive (S) model (see Equation 3) isolates $I_{\mathrm{KLT}}$ using a non-inactivating sodium current by freezing the inactivation gating variable of the RM03 model at its resting state value. Without dynamic $\mathrm{Na}^{+}$inactivation, the $\mathrm{S}$ model can develop bistability (coexistence of $V_{\text {rest }}$ with a stable depolarized state). To prevent such bistability and preserve type III excitability we reduce $\bar{g}_{\mathrm{Na}}$ from 1000 to $177 \mathrm{nS}$ (see Appendix B in Supplementary Material for more details). Although this is a strong reduction (by a factor of $1 / 5$ or so), notice that $\mathrm{Na}^{+}$ strongly inactivates at subthreshold values of voltage and spikes ocurr with $h$ approximately 0.1 , thus the total $\mathrm{Na}^{+}$conductance at spike onset is more comparable. Nevertheless, even with this reduction, the density of sodium channels for the $S$ model is sufficient to amplify subthreshold synaptic potentials, thus retaining the excitability property. The divisive (D) model (see Equation 6) isolates $\mathrm{Na}^{+}$inactivation by freezing the potassium activation variable at its resting value $\left(w_{0}=w_{\infty}\left(V_{\text {rest }}\right)\right)$. However, freezing $w$ to its resting value converts this reduced $\mathrm{D}$ model (as well as the original RM03) from phasic to tonic (Day et al., 2008; Gai et al., 2009, 2010; Meng et al., 2012). To counteract this effect and retain phasic firing in the D model we shift $h_{\infty}$ by $6 \mathrm{mV}$ leftwards with respect to the original $h_{\infty}$ in RM03 (see Figure 1). Moreover, in order to better compare the results with the S model we decrease $\bar{g}_{\mathrm{Na}}$ from $1000 \mathrm{nS}$ to $500 \mathrm{nS}$. Finally, we combine these two mechanisms in a 3-variable model: the combined (C) model (see Equation 9) has $I_{\mathrm{KLT}}$ of the $S$ model and $I_{\mathrm{Na}}$ of the $\mathrm{D}$ model.

\subsection{Dynamic Properties for Different Type III Mechanisms}

Each of the models, C, D, S, fires phasically (only one spike, if any, at the onset of the input; see Figure 2A, inset) and its steady state $V_{s s}$ depends on $I$, the applied current, but is stable for any $I$ (Figure 2A). There is no bifurcaton to repetitive firing on the $V_{s s}$ vs. I curve - a signature of type III excitability. For a slow modulation of the input current $I$ there is no spiking; the voltage tracks the $V_{s s}$ vs. I relation. Depolarization in the $\mathrm{S}$ and $\mathrm{C}$ models with $I$ is modest because of $I_{\mathrm{KLT}}$ activation. In contrast to this rectification in the $V_{s s}$ vs. I relation for $\mathrm{S}$ and $\mathrm{C}$, the $\mathrm{D}$ model has a nearly linear relation without $V$-gating of $I_{\mathrm{KLT}}$ and negligible steady state $I_{\mathrm{Na}}$. Thus, the set of voltages at steady state spans a wider range for the $\mathrm{D}$ model than for the $\mathrm{S}$ and $\mathrm{C}$ models.

In a phasic firing system, spikes are elicited only for transient inputs, fast enough and strong enough inputs. For step current input our models respond sensitively to a step's amplitude (Figures $\mathbf{2 A}$ (inset),C), effectively showing a threshold-like behavior for this stimulus class. The $\mathrm{C}$ model has higher current threshold than the D model - they have the same $\bar{g}_{\mathrm{Na}}$ but $\mathrm{C}$ has two negative feedback processes. $\mathrm{S}$ has the highest current threshold, reflecting the decreased $\bar{g}_{\mathrm{Na}}$. Notice that the amplitude of spikes is modulated accordingly.

Phase plane analysis and relative time scales between voltage and gating variable help us understand dynamic features of spike generation (see Figure 2B and Methods Section). The trajectories corresponding to the time courses in Figure 2A (inset) reveal the characteristics of excitability: amplification of $V$ for an adequate 
stimulus and then recruitment of dynamic negative feedback (Figure 2B). For these phasic systems, the steady state is always on the "left branch" for each $I$-value, guaranteeing its stability (Rinzel and Huguet, 2013). For steady strong input the $V$ nullcline's cubic or $\mathrm{N}$-shape is greatly diminished corresponding to suppressed excitability (strongly activated $I_{\mathrm{KLT}}$ or strongly inactivated $I_{\mathrm{Na}}$ ). Notice that this change is more dramatic in the $\mathrm{D}$ model than in the $\mathrm{S}$ model (the $V$-nullcline for $I=0.6 \mathrm{nA}$ in the $\mathrm{D}$ model is hardly visible).

When the external input $I$ increases, the steady state drifts downward along the left branch and the phase point approaches the steady state. In the $S$ model, activation of $I_{\text {KLT }}$ precludes excessive depolarization, limiting the steady state voltage to values no higher than -60 to $-50 \mathrm{mV}$. Close to the resting state, the voltage and the gating variable have "comparable" timescales (within a factor of 3), and the phase point does not escape rightward directly (red trajectories in Figure 2B). Only for large enough input $I$, the phase point can enter the region with a clear time-scale separation (blue region for values of $V$ above -40 $\mathrm{mV}$ ), and escape rightward toward the right branch of the $V$ nullcline, generating a spike (green trajectories in Figure 2B). Repolarization of the membrane potential after a spike is only due to the Ohmic leak current in the D model, unlike S and C models that have the $V$-gated potassium current, and there is no hyperpolarization when the spike terminates.

For a time-varying input the $V$-nullcline and target steady state move with the stimulus. Thus, for a slow stimulus ramp, the phase point tracks slowly the drifting steady state and there is no spike. For a fast enough ramp the phase point can escape rightward and lead to a single spike and then settle to a stable depolarized steady state. For this reason, phasic neurons are often called differentiators because they respond to fast-rising, but not to slow-rising, inputs (Ferragamo and Oertel, 2002; Svirskis et al., 2002; McGinley and Oertel, 2006; Izhikevich, 2007; Ratte et al., 2014). Our D model is a less sensitive differentiator; its critical value for the ramp slope to elicit an action potential is lower (see Figure S1A) and, in response to a half-wave rectified sinusoidal input (Equation 10), it can fire at lower frequencies than $\mathrm{C}$ and $\mathrm{S}$ (see Figure S1B).

\subsection{Type III Excitability is More Robust in the Presence of $\mathrm{Na}^{+}$Inactivation}

Type III excitability occurs in each of our representative models but more or less robustly for mixtures of $I_{\mathrm{Na}}$ and $I_{\mathrm{KLT}}$. We identified regions in the parameter space $\left(\bar{g}_{\mathrm{Na}}, \bar{g}_{\mathrm{KLT}}\right)$ where the models behave phasically, i.e. the fixed point is stable for any value of $I$ (Figure 3A, gray region). For other $\bar{g}_{\mathrm{Na}}-\bar{g}_{\mathrm{KLT}}$ combinations the models show destabilization of the fixed point through a Hopf bifurcation and repetitive firing (Figure 3A, red region) and the possiblity of multiple steady states, including a stable steady state of high voltage, "lockup," (Figure 3A, white region).

The divisive mechanism is key to guarantee the robustness of the phasic properties of the system with respect to changes in channel density. Removal of $\mathrm{Na}^{+}$inactivation, as in the $\mathrm{S}$ model, strongly constrains the parameter range for type III excitability. To avoid "lockup" in the S model (large white region in Figure 3A) we chose its value of $\bar{g}_{\mathrm{Na}}$ smaller than for D and

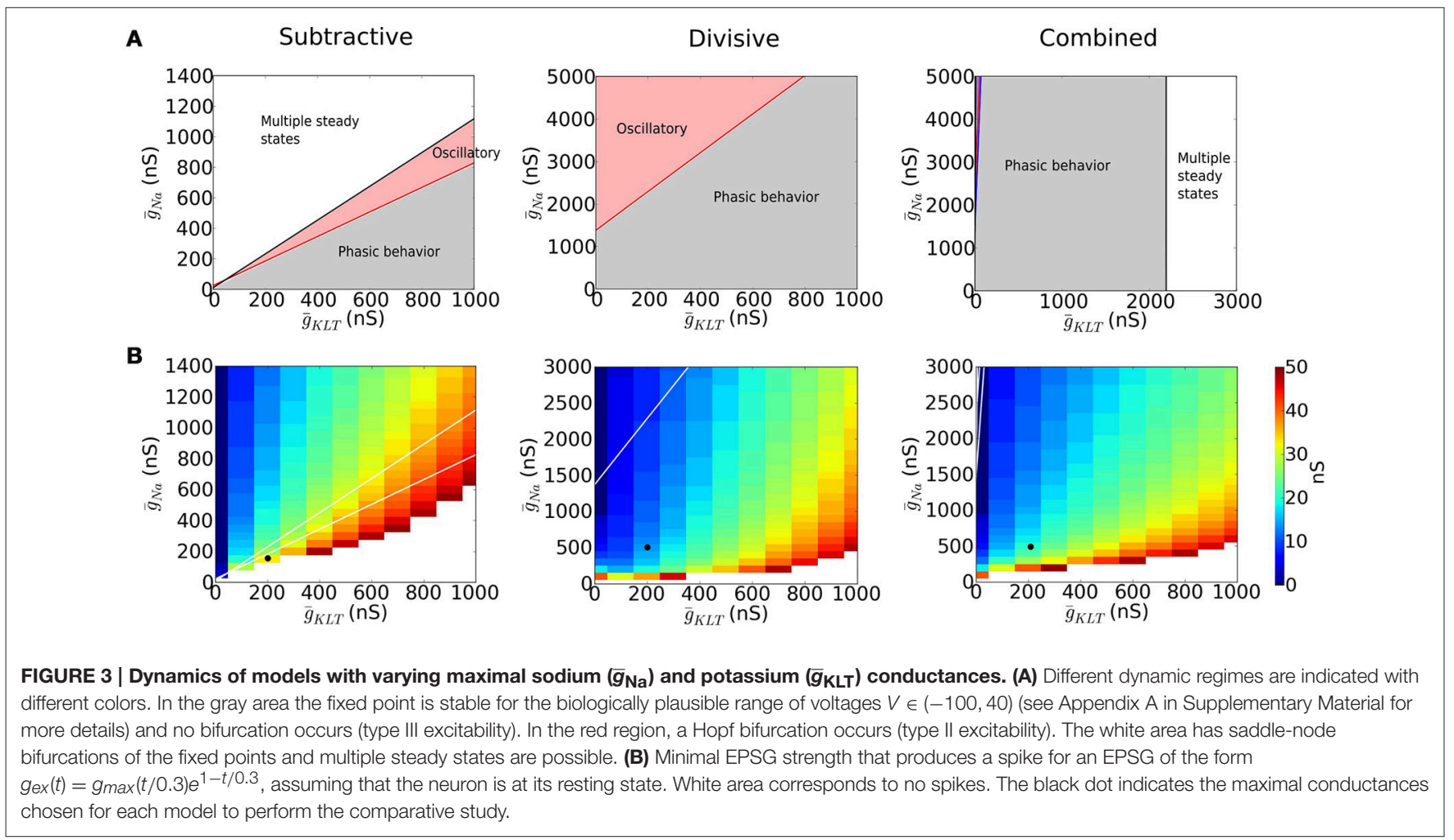


$\mathrm{C}$ models; consequently, a stronger input was required for the $\mathrm{S}$ model to be excited (see Figure 2C). By comparing the parameter spaces of the D and C models, we observe that when $\bar{g}_{\mathrm{Na}}$ in the $\mathrm{D}$ model is increased, the system transitions to a regime with oscillatory behavior, while the presence of $I_{\mathrm{KLT}}$ narrows this region (compare Figure $\mathbf{3 A}$ for $\mathrm{D}$ and $\mathrm{C}$ ).

The threshold for eliciting a spike with a brief synaptic excitatory conductance input (EPSG) depends on $\bar{g}_{\mathrm{Na}}$ and $\bar{g}_{\mathrm{KLT}}$ (see Figure $3 \mathrm{~B}$ ). If $\bar{g}_{\mathrm{Na}}$ is small, then the system is not excitable (white region in Figure 3B). Of course, as $\bar{g}_{\mathrm{Na}}$ increases the system becomes more excitable and the EPSG threshold decreases. Moreover, if $\bar{g}_{\mathrm{KLT}}$ is large, more inward current ( $I_{\mathrm{Na}}$ plus the excitatory current) is needed to overcome the opposing potassium current and EPSG threshold increases. Thus, in Figure $3 \mathbf{B}$, along contours, $\bar{g}_{\mathrm{Na}}$ increases as $\bar{g}_{\mathrm{KLT}}$ increases.

\subsection{Non-monotonic $f$-I Curves for Noisy, Constant Mean Input Current}

Neurons and models with type III excitability respond to input transients but not to slowly varying inputs. In contrast, type I and type II excitable systems fire repetitively for steady input current. Their input-output relations, firing frequency vs. current ( $f$ - $I$ curve), typically show monotonic increase over much of the $I$-range and then at high $I$-values loss of oscillation with either gradually decreasing amplitude or with a sudden drop in frequency and amplitude (Rinzel and Ermentrout, 1998; Borisyuk and Rinzel, 2005; Rinzel and Huguet, 2013). For type III excitability, an $f-I$ relation quantifies the response to a fluctuating input: the mean firing frequency vs. the mean of a noisy current. The $f-I$ curve is non-monotonic, as seen in some illustrative cases (Higgs et al., 2006; Lundstrom et al., 2008, 2009; Gai et al., 2009), and particularly distinctive from typical (noise free) type I and II cases. The firing frequency (firing probability per unit time) decreases smoothly toward zero for high mean $I$ and the $f$ - $I$ relation shows strong sensitivity to input variance.

Our three phasic models have non-monotonic $f-I$ relations and show strong sensitivity to noise (Figure 4A). The $f$ - $I$ curves are similarly shaped for the $\mathrm{S}$ and $\mathrm{C}$ models but less symmetric than D's $f$ - $I$ curves. There is no repetitive firing without noise; the increase of firing probability with noise level evidences the models' sensitivity to input transients (different colors in Figure 4A). The $\mathrm{S}$ model fires less frequently than $\mathrm{D}$ or $\mathrm{C}$ to a given noise level, partly because $\bar{g}_{\mathrm{Na}}$ is smaller for the $\mathrm{S}$ model requiring stronger fluctuations to generate spikes (see Figure 3B). The $\mathrm{C}$ model shows intermediate sensitivity to noise.

We highlight the different effects of subthreshold $V$ dependent conductance gating by replotting the input-output relations of Figure 4A in terms of mean voltage, $\langle V\rangle$ (Figure 4B). For $\mathrm{S}$ and $\mathrm{C}$ the maximum firing rate occurs for $\langle V\rangle$ around $V_{\text {rest }}$, slightly depolarized for $\mathrm{D}$. The firing rate of $\mathrm{S}$ and $\mathrm{C}$ decreases for increased $\langle V\rangle$ and falls abruptly to zero for $\langle V\rangle$ just below $-50 \mathrm{mV}$, less than the activation voltage for $I_{\mathrm{Na}}$. This abrupt falloff reflects the shunting effect of strongly activated $g_{\text {KLT }}$ and reduced amplitude of $V$ fluctuations. On the other hand, firing rate rises gradually with $\langle V\rangle$ below $V_{\text {rest }}$ with substantial firing probability $\langle V\rangle$ well below $V_{\text {rest }}$. At these hyperpolarized levels the input resistance is high ( $g_{\mathrm{KLT}}$ is deactivated) and $V$-fluctuations are substantial. In contrast to the asymmetry in the $f-V$ relations for $\mathrm{S}$ and $\mathrm{C}$ the relation for $\mathrm{D}$ is more symmetric, like that for $f-I$ relation of $\mathrm{D}$. Ohmic leak is the only conductance at the foot and tail of the $V$-range for $\mathrm{D}$. Therefore, the input resistance and voltage fluctuations are comparable.

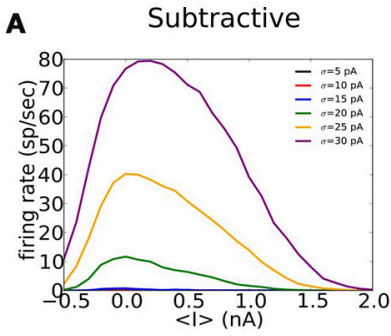

B

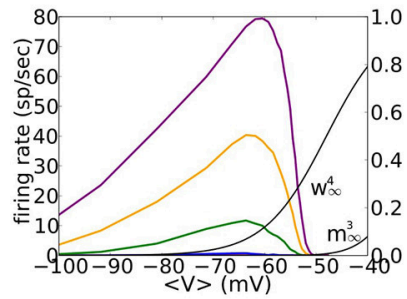

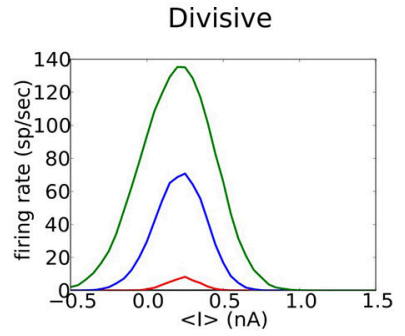

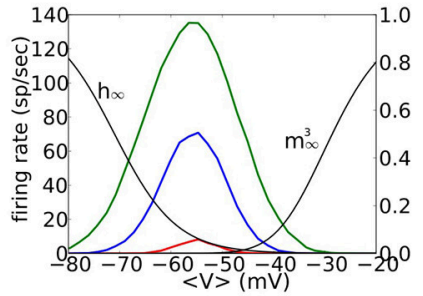

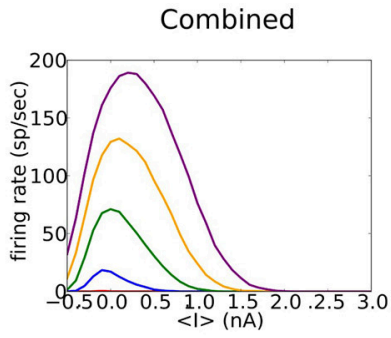

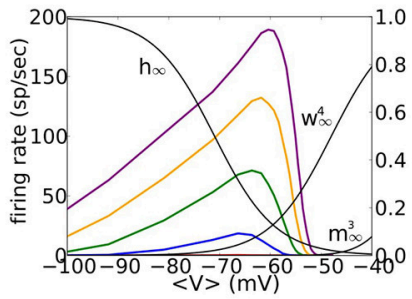

FIGURE 4 | Firing rate for noisy input current, as a function of mean input and mean voltage shows non-monotonic behavior. (A,B) Inputs to the $S$ (left), D (middle), and C (right) models are noisy currents modeled as Gaussian white noise with constant nonzero mean $\langle I\rangle$ and noise intensity $\sigma=5,10,15,20,25,30$ pA (different colors shown in legend). (A) Output firing rates vs. mean input current $\langle I\rangle$. (B) Output firing rates vs. mean voltage $\langle V\rangle$. Solid black thin curves are $h_{\infty}, m_{\infty}^{3}$, and $w_{\infty}^{4}$ used for each model. Mean voltage $\langle V\rangle$ are computed from the time series by ignoring a 4 ms time window that surrounds a spike. 


\subsection{Non-monotonic Input-output Curves for Stochastic Synaptic Input}

The noisy current input of Section 3.4 may be viewed as an idealization of random synaptic input delivered to a cell. Here, we consider the response to trains of excitatory synaptic conductance inputs (EPSGs). The event times are Poisson distributed and the EPSG amplitudes are binomially-distributed (see Section Methods) with mean amplitude that will just elicit a spike from the resting state. The three models show nonmonotonic input-output curves, firing rate vs. EPSG input rate (Figure 5A, black curves), qualitatively as seen in Figure 4A for noisy current injection but some features differ. On the low input side the models fire for current injection (Figure 4A) even for non-positive $\langle I\rangle$, since by chance some positive current fluctuations are strong enough to cause a spike. But for synaptic input, firing only occurs for positive EPSG rate (Figure 5A). This feature is also seen in Figure 5B (black curves) where the firing rate for stochastic EPSG input drops abruptly to zero as $\langle V\rangle$ decreases below $V_{\text {rest }}$; this corresponds to zero EPSG rate, i.e. no synaptic input. Substantial mean input, either noisy current injection or stochastic ESPGs, leads to depolarization and reduced firing probability. But for synaptic input, high EPSG rate leads to increased membrane conductance, thus EPSGs that might be superthreshold for lower input rates are not for higher rates; the EPSG threshold has effectively increased thereby attenuating the effect of input variance and smoothing the drop in firing rate (see more details in Figure S2). For the D model, the conductance nature of synaptic input accounts for the loss of symmetry in its input-output relation.

Synaptic inhibition differently affects our three models while they are undergoing stochastic EPSG input. For the $S$ and C models, not surprisingly (although, see below), inhibition decreases the firing rate for a given EPSG rate (Figure 5A, red and green curves). In contrast, for the D model the relationship between firing rate and inhibition can be non-monotonic (Figure 5A), depending upon the EPSG rate. Specifically, the firing rate decreases with $g_{\text {inh }}$ for low EPSG rate, whereas for high EPSG rate firing rate increases. Consequently, at moderate input rates the firing rate behaves non-monotonically, increasing then decreasing with $g_{\text {inh }}$ (see more details in Figure S3).

There are some notable features of the input-output relations, firing rate vs. $\langle V\rangle$ (Figure 5B). Overall, the relations appear qualitatively similar for the 3 models, non-monotonic with a sharp rise and gradual fall for increasing $\langle V\rangle$. The "left branch" extends to lower $\langle V\rangle$ with $g_{\text {inh }}$; this is because $g_{\text {inh }}$ lowers the effective resting voltage, where firing rate descends to zero as 〈EPSG〉 rate decreases to zero. For the $\mathrm{S}$ and $\mathrm{C}$ models, in case of either stochastic EPSGs, here (Figure 5B), or for noisy current injection (Figure 4B), firing rate decreases from its maximum to near zero for the same $10 \mathrm{mV}$ range: $(-60,-50 \mathrm{mV})$. Notably, the maximum firing rate occurs at the same mean voltage independently of the inhibition level; this "sweet spot" for firing is again located about the same voltages as for the noisy input (Figure 4B): $\sim-60 \mathrm{mV}$ for $\mathrm{S}$ and $\mathrm{C}$ and at a higher depolarized state $(\sim-55 \mathrm{mV})$ for $\mathrm{D}$. From the perspective of a given $\langle V\rangle$
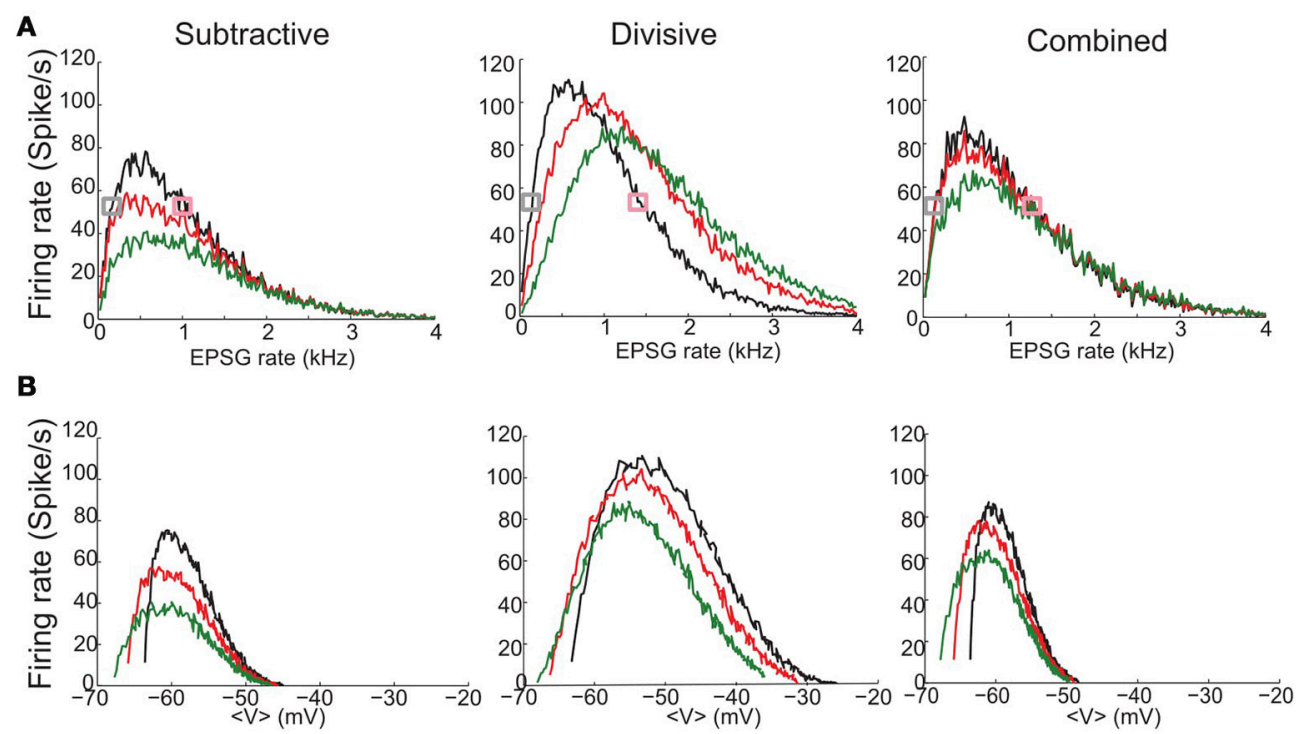

FIGURE 5 | Firing rate for stochastic synaptic inputs as a function of EPSG input rate and mean voltage depolarization shows different responses for constant inhibitory synaptic inputs. (A,B) Inputs to the S (left), D (middle), and C (right) models are trains of excitatory synaptic conductance inputs (EPSGs) that are Poisson distributed in time and binomially-distributed in amplitude (see Section Methods). The mean amplitude is different for each model and is chosen according to the threshold to elicit a spike from the resting state: $8 \mathrm{nS}$ (S model); $3.86 \mathrm{nS}$ (D model) and $5.7 \mathrm{nS}$ (C model). (A) Output firing rate as a fucntion of EPSG input rate ( $x$-axis) in the absence of inhibition (black) and under the presence of a synaptic inhibitory current of constant conductance: $g_{\text {inh }}=10$ (red), 20 nS (green). (B) Output firing rate obtained in $(\mathbf{A})$ plotted a function of mean membrane potential $\langle V\rangle$. Mean voltages $\langle V\rangle$ are computed from the time series by ignoring a $4 \mathrm{~ms}$ time window that surrounds a spike. Curves are colored according to the inhibition strength as in (A). Figure S3 shows output firing rate for a larger range of inhibitory strengths. Figure S2 shows spike triggered averages for the simulation runs indicated with gray and pink squares in (A). 
we see (except for $\langle V\rangle$ less than, say, $-60 \mathrm{mV}$ ) that the firing rate decreases with $g_{\text {inh }}$. The effect is monotonic decreasing in each model. The reason being that in order to maintain a fixed $\langle V\rangle$ as $g_{\text {inh }}$ increases, the excitatory input, EPSG rate, must also increase. That is, to keep a balanced state of fixed $\langle V\rangle$ both synaptic excitation and inhibition increase or decrease together. The amount of increased $g_{i n h}$ that compensates for increased excitatory input to maintain a balanced $\langle V\rangle$ is smaller for the $\mathrm{D}$ model than for $\mathrm{S}$ and $\mathrm{C}$ due to D's larger input resistance (see Figure S3).

\subsection{Precision and Detection of Coincident Inputs for Periodic Drive \\ 3.6.1. Coincidence Detection, Reliability, and Precision of Entrainment}

Neuron models with type III excitability are good coincidence detectors, as are neurons in the auditory brainstem. That is, they respond when multiple inputs arrive tightly-timed in a volley but not if the same inputs are dispersed in time. Moreover, such neurons can phase lock with high precision to an external periodic stimulus; they fire precisely timed on each stimulus cycle, sometimes enhancing the input precision (Joris et al., 1994; Joris and Smith, 2008).

We adopt a stochastic input framework to characterize the models' coincidence detection properties for cyclic drive. Each cycle has a fixed number of inputs; they are more or less time coherent for higher or smaller values of $b$, respectively, according to a von Mises distribution (Figure 6 and Methods). The probability of firing in a cycle varies with the input frequency and temporal coherence of inputs (Figure 7). The V-shape of these parameter regimes for firing is not unexpected. They resemble the diagrams for phase-locking of the models for periodic inputs, as for full or partially rectified sinusoidal current injection (Figure S1B and Gai et al., 2009, 2010; Meng et al., 2012). However, for deterministic periodic input the transition in input parameter values from 1:1 entrainment to low firing rate is sharp at low frequencies, here it is gradual because the composite input per cycle involves stochastically-timed unitary events. The curved nature of the high and low frequency legs of the $\mathrm{V}$ reflect the threshold of these models for an input's rising slope together with the feature that temporal coherence here is with respect to a cycle's phase not absolute time. Say, the need for higher $b$ values to ensure firing for lower frequency inputs accounts for the need to have fast rising input. We also see here that the V's apex, around $200-300 \mathrm{~Hz}$, is similar to that of S and C for sinusoidal input, suggestive of a resonant like property of these models (Remme et al., 2014). These models do not fire for our EPSG volleys for cyclic frequency above $400 \mathrm{~Hz}$, regardless of the input temporal coherence $b$. Temporal summation of the inputs for $\tau_{s}=0.3 \mathrm{~ms}$ leads to enough baseline activation of negative feedback to preclude spiking. This upper limit of frequency is larger if unitary inputs are larger (compare Figures 7A,B). Later, we will see that inhibition may restore the excitability properties
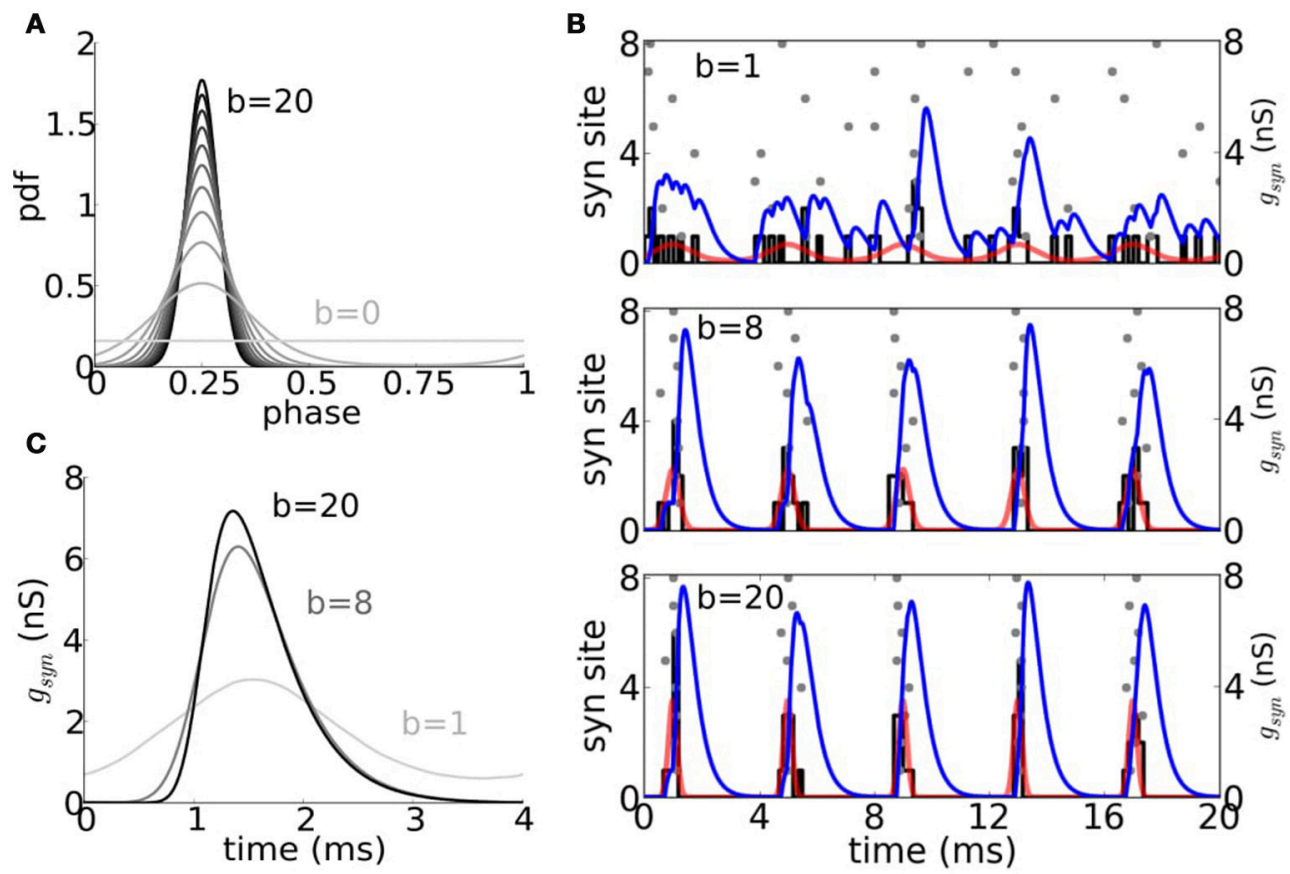

FIGURE 6 | Periodic trains of composite excitatory synaptic inputs with random event times. (A) Probability density function (pdf) of the von Mises distribution for different values of the temporal coherence parameter $b$ ranging between 0 and 20 in increments of 2 units. (B) Raster plot of event times per site (8 sites) drawn from a von Mises distribution (pdf shown in red) with $b=1$ (top), $b=8$ (middle), and $b=20$ (bottom), together with the input period histogram (black) and composite EPSG (blue curve) obtained from the summation of individual mEPSG with $g_{\max }=1$ and $f=250 \mathrm{~Hz}$ (see Section Methods). (C) Averaged composite EPGS for $b=1, b=8$, and $b=20$. 

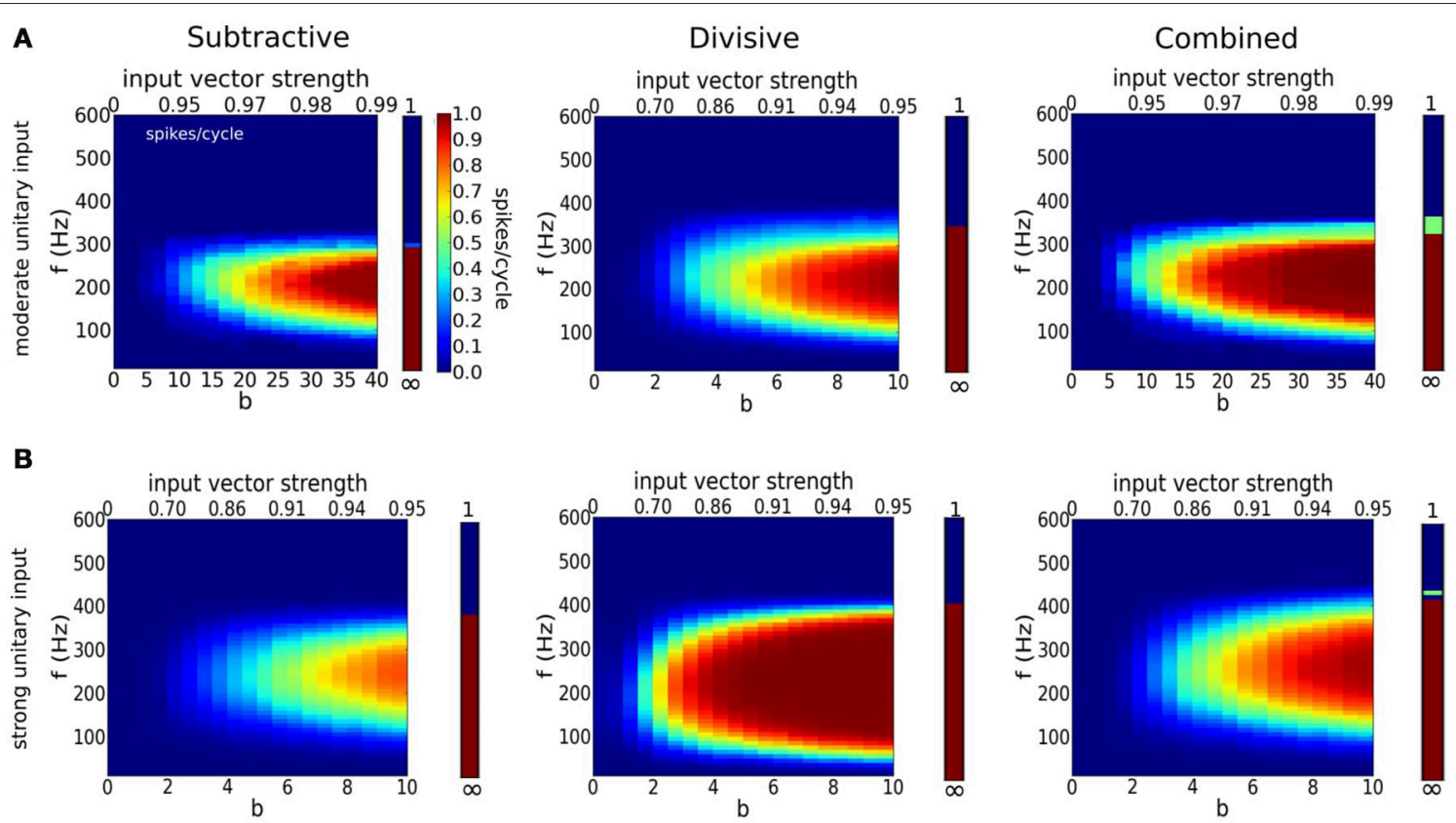

FIGURE 7 | Detection of coincident inputs for a periodic train of multi-synaptic inputs with varying temporal coherence and frequency. (A,B) Firing probability per cycle $(0<$ spikes/cycle $<1)$ for $S$ (left), D (middle), and $\mathrm{C}$ (right) models as a function of temporal coherence in the arrival of inputs ( $x$-axis; bottom axis shows temporal coherence parameter $b$ in von Mises distribution, while top axis shows its corresponding input vector strength) and input frequency $f$ ( $y$-axis). Bars on the right hand side of panels correspond to firing rate when dispersion is null $(b=\infty)$. Notice that temporal coherence $b$ refers to a cycle, thus the dispersion of inputs in absolute time for a given value of $b$ decreases with input frequency. (A) Moderate individual EPSGs (only 6 or more coincident inputs exceed threshold for spike from the resting state): $5 \mathrm{nS}(\mathrm{S}), 2.5 \mathrm{nS}(\mathrm{D}), 3.5 \mathrm{nS}(\mathrm{C})$. (B) Strong individual EPSGs (only 4 or more coincident inputs exceed threshold for spike from the resting state): $7.5 \mathrm{nS}(\mathrm{S}), 3.75 \mathrm{nS}$ (D), 5.25 (C). Computations were performed with 1000 cycles.

in these situations, especially for the D model. Note that for the sinusoidal input of Figure S1B we allowed amplitude to be a stimulus parameter and therefore entrainment could be to very high frequencies.

For both moderate and strong inputs, (Figures 7A,B, respectively), the $\mathrm{D}$ model is less selective to time coincident inputs. The $\mathrm{D}$ model can spike with higher probability for weak temporal coherence than the S and C models; D has a lower threshold for input slope that causes firing (see Figure S1B). The $\mathrm{D}$ model's lower slope threshold is further seen in D's tolerance for low frequency inputs. The more stringent requirement of $S$ and $\mathrm{C}$ for steeper input slope is also seen in the spike triggered averages (see Figure S4). For weakly coincident inputs (small $b$ ), only a few realizations lead to composite EPSGs that are steep enough to cause firing, hence the low firing probability for $\mathrm{S}$ and $\mathrm{C}$ models. This observation suggests that models with dynamic $I_{\mathrm{KLT}}$ are more sensitive to inputs arriving together while the $\mathrm{D}$ model, with frozen conductance for $I_{\mathrm{KLT}}$ is far less selective about how these inputs are distributed along a cycle.

The firing probability increases for each of the models if the unitary input strength increases (compare Figures 7A,B). Coincidence sensitivity persists with incresing input strength although the minimum degree of coincidence needed for firing decreases (leftward shift of colored region). The stronger unitary inputs lead to larger increments in rising slope per event. It is easier to generate with fewer inputs a fast enough rising composite input to cause a spike. The $\mathrm{D}$ model here as well fires less selectively than S and C; at 50\% rate for very weak coincidence ( $b=2$, with $\mathrm{VS} \sim 0.7)$ over a substantial input frequency range. This feature of the $\mathrm{D}$ model is likely reflecting the passive and slower time course of membrane potential decay after a subthreshold EPSP peak, in contrast to the active decay from $I_{\mathrm{KLT}}$ in the S and C models (Jercog et al., 2010; Khurana et al., 2011; Mathews et al., 2010). This allows more time for a subsequent EPSG to give another sharp rise to the input. The D model, although legitimately phasic by our definition, behaves somewhat like an integrator for summation of fast unitary inputs.

It is rather striking to notice that, for a fixed input frequency (say, $250 \mathrm{~Hz}$ in Figure 7A), the dependence of firing probability on $b$ for the $\mathrm{S}$ and $\mathrm{C}$ models is considerably more gradual than for the $\mathrm{D}$ model. For $b=8$ less than $50 \%$ of inputs rise fast enough to cause $S$ to fire; for $b=20$ about $70 \%$ are fast enough (see Figure 6B). For very large $b(\sim 35)$ the firing probability becomes close to one. Only when the event times are very tightly timed will the input's rise be treated as fast enough for S. For lower values of $b$ the fraction of realizations (cycles) that have events so tightly timed is less and this dependence on $b$ is gradual for $\mathrm{S}$ and $\mathrm{C}$. The range of $b$ values for which the firing rate rises from 0 to 1 can be considered as the dynamic range for coincidence detection. Although this dynamic range is wide for $\mathrm{S}$ and $\mathrm{C}$ the slope threshold is high and demands for detecting coincidence in a cycle's composite input are high. The $\mathrm{S}$ and $\mathrm{C}$ 


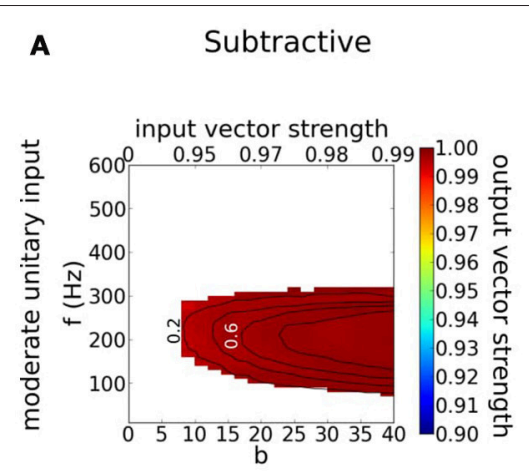

Divisive Combined
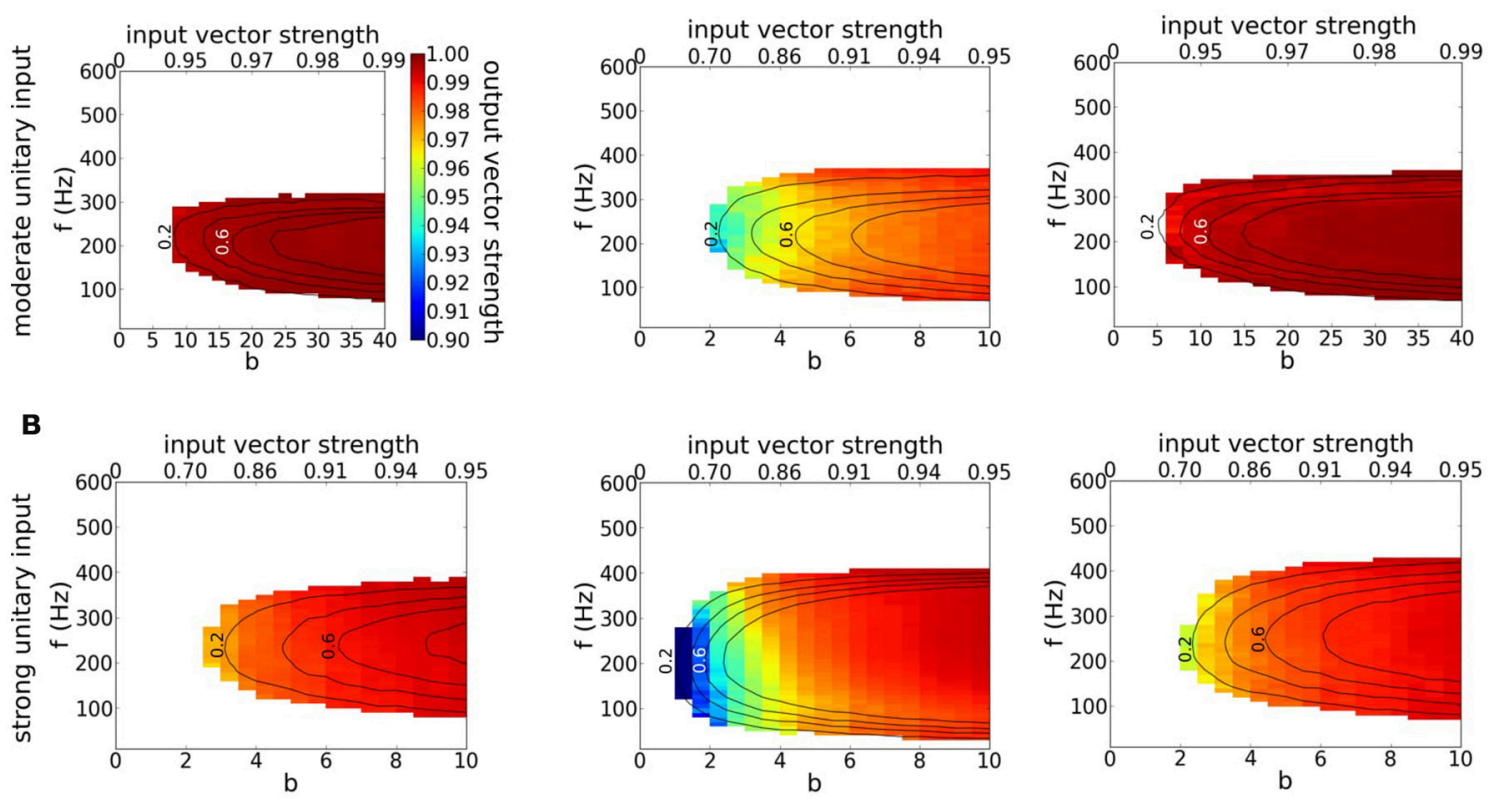

FIGURE 8 | Precision of phase-locking to a periodic train of multi-synaptic inputs with varying temporal coherence and frequency. (A,B) Output vector strength for simulation runs in Figure $\mathbf{7}$ for the $\mathrm{S}$ (left), D (middle) and $\mathrm{C}$ (right) models as a function of temporal coherence in the arrival of inputs ( $x$-axis; bottom axis shows temporal coherence parameter $b$ in von Mises distribution, while top axis shows its corresponding input vector strength) and input frequency $f(y$-axis). (A) Moderate individual EPSGs. (B) Strong individual EPSGs. Contours correspond to output firing rate probability per cycle of 0.2, 0.4, 0.6, and 0.8 (spikes/cycle).

models with a large dynamic range have a firing probability that encodes the coincidence. The $\mathrm{D}$ model is less discriminatory. It will fire at high probability for far weaker coincidence and its firing probability is not well graded.

Each model phase-locks with high precision to the cyclic stimulus, as seen by the high vector strength, VS is above 0.9 in most of the area where responses exceed 0.1 spikes/cycles (Figure 8, non-white region). Interestingly, the output VS is substantially higher than the input VS (Figure 8, top horizontal axis), evidencing that these coincidence detectors can enhance the precision of entrainment. Although the three models seem quite similar in terms of VS, they differ significantly in terms of histogram shape (Figure 9C). Thus, models with dynamic $I_{\mathrm{KLT}}$, when they respond, they do so at a higher precision than the $\mathrm{D}$ model. This fact is especially noticeable at low input strength (see Figure 8A).

\subsubsection{Coincidence Detection and Precision of Entrainment in the Presence of White Noise}

Our coincidence detector models show varied responses in the presence of background (white) noise to inputs like those of Figure 7. For conditions of low firing probability (low $b$ and low to moderate input frequency) the noise can boost subthreshold inputs and induce firing, thereby increasing detectability. However, for conditions of high (noise free) firing probability, noise reduces firing probability and some suprathreshold inputs go undetected-reliability is decreased. We found that precision is reduced by noise for all three models, but especially for $\mathrm{D}$. We illustrate these effects for input at frequency $250 \mathrm{~Hz}$ with low unitary input strength (as in Figure 7A). For a given noise level, firing probability increases with $b$, initially, above the noise free probability but ultimately below (Figure 9A). As noise strength increases, the three models show an increased firing rate, for low to moderate $b$-values. The $\mathrm{S}$ and $\mathrm{C}$ models retain some gradation of firing rate with degree of coincidence, $b$, but the $\mathrm{D}$ model has a much sharper rise to saturation, thus compromising the rate encoding of coincident inputs. Moreover, the minimum value of $b$ for spiking is substantially diminished in the $\mathrm{D}$ model; it fires spontaneously at high noise levels. The precision (measured as VS) decreases with noise in all models but far more for the D model than for S or C (Figure 9B).

The $\mathrm{S}$ and $\mathrm{C}$ models entrain with different phases than does $\mathrm{D}$ although noise induces more firing during the rising slope of the mean EPSP for each model in the low to moderate firing rate regime. Consider the case of $250 \mathrm{~Hz}$ input, as in Figures 9A,B. In the noise free case the firing times for the three models occur mostly at the peak of the EPSP for the average synaptic conductance (Figure 9C). With noise (10 pA here), the firing time distribution spreads more toward earlier firing corresponding to the rising phase of the EPSP, and this effect is more noticeable in the D model (Figure 9D). Most of the added spikes thus appear to reflect slope sensitivity, enhanced by the noise. This effect is reminiscent of earlier findings (Gai et al., 2009,2010 ) in which firing to slow modulation could be induced on the rising phase when no firing occurred in the absence of noise, a feature referred to as slope-based stochastic resonance. 


\section{Noise intensity increases with line intensity}

A

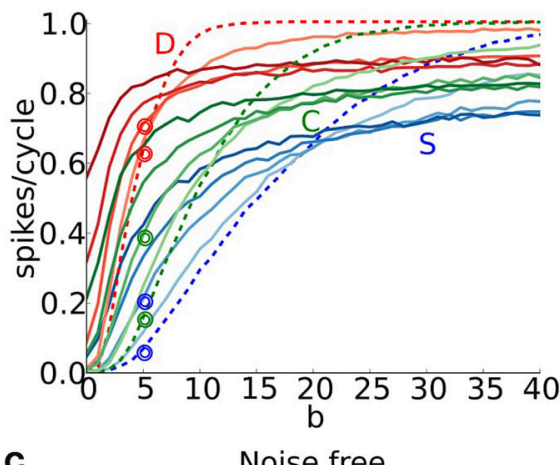

c

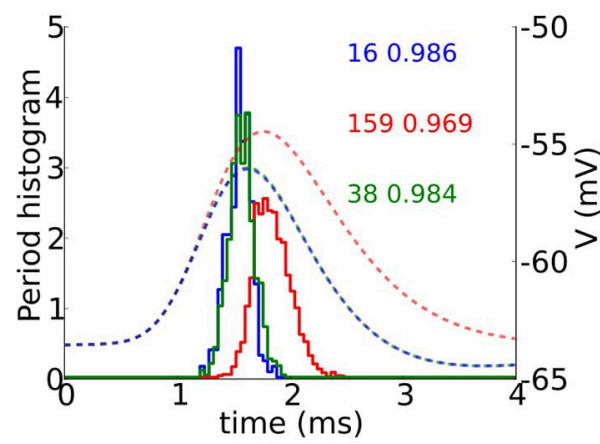

B

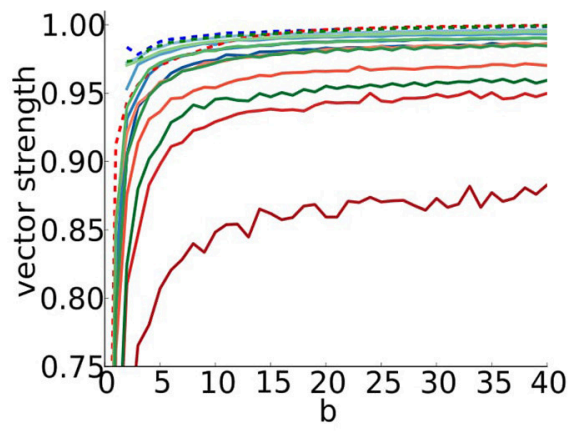

D

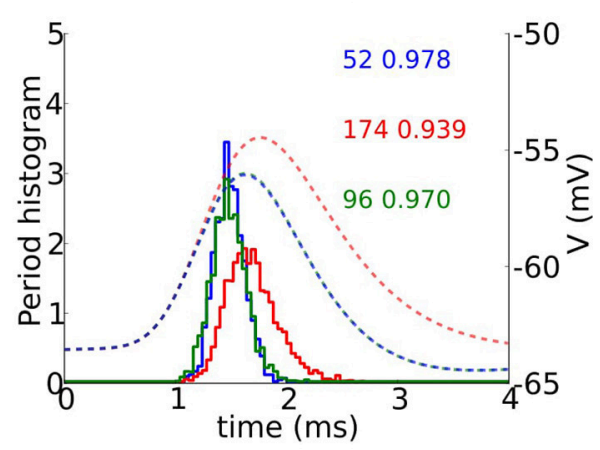

FIGURE 9 | Entrainment and vector strength properties for a periodic train of multi-synaptic inputs in the presence of background (white) noise.

(A) Output firing rate (spikes/cycle) and (B) vector strength for S (blue), D (red), and C (green) models for a periodic input of $f=250 \mathrm{~Hz}$ and low unitary vector strength ( 6 coincident inputs exceed threshold for spike from the resting state, as in Figures 7A, 8A) as a function of input temporal coherence $b$ for different noise intensities: dashed line corresponds to the noise free system and color intensity indicates noise intensity ranging from 5 to 20 in steps of 5. (C,D) Period histograms (solid curves) for S (blue), D (red), and C (green) models for responses to inputs indicated with empty circles in (A). (C) Corresponds to the noise free system and (D) to noise strength $10 \mathrm{pA}$. We show EPSPs (dashed curves, notice that blue and green lie on top of each other) for the average composite EPSGs obtained from the summation of individual $\mathrm{mEPSG}$ with $g_{\max }=1$ (take note that this is not the maximal amplitude used in simulations). Numbers indicate firing rate (left) and vector strength (right) for the different models.

Here, the spread of firing times carries the consequence of degraded precision, a trade-off for enhanced slope sensitivity. The D model is more vulnerable to this phenomenon due to its input resistance. We see here that small differences in noise (and thus input vector strength) translate into significant effects on the period histogram's shape, and on the firing rate for $\mathrm{S}$ and $\mathrm{C}, 2-3$ times increased.

\subsubsection{The Timing of Brief Inhibition Can Counter or Promote Firing}

Inhibition can have contrasting effects, negative or positive, on firing probability. We showed such contrasts for steady inhibition $\left(g_{\text {inh }}\right)$ and Poisson distributed excitatory synaptic input events (Figure 5). For a substantial range of EPSG input rates, increasing $g_{\text {inh }}$ leads to decreased firing probability an expected outcome. However, for high EPSG input rates that lead to substantial reduction in excitability (the nonmonotonic input-output feature of type III excitability), we found that $g_{\text {inh }}$ can promote firing for the D model by decreasing membrane potential and thereby partially restoring excitability. Here, for repetitive excitatory input we show that brief inhibition may increase or decrease firing probability depending on the timing of inhibition and, further, that the promoting effect may be enhanced if inhibition is more sharply timed (higher $b$-value).

The familiar phenomenon of post-inhibitory rebound relates to the hyperexcitability following release from a sufficient duration of hyperpolarization that can cause a spontaneous spike or induce one from a subthreshold depolarizing input. Perhaps more surprising is that a brief, well-timed, hyperpolarizing inhibition can have a similar effect, leading to a spike for an otherwise subthreshold EPSG. This phenomenon, referred to as Post-Inhibitory Facilitation (PIF), has been demonstrated for the Hodgkin-Huxley model (Dodla and Rinzel, 2006) (Type II excitability) as well as for auditory brain stem neurons and the RM03 model (Dodla et al., 2006) (Type III excitability). Here, we characterize and compare the timing window, intensity range and VS range for reduction and facilitation of firing probability for the $S, D$, and C models. The excitatory stimulus is a repetitive von Mises distributed event train as we used for characterizing features of entrainment (Figures 6-8). Each cycle includes an inhibitory input (von Mises) with a specified phase difference 
with respect to excitatory input (they are coincident at phase 0 , see Section Methods). We will consider two different input frequencies: a low frequency $(150 \mathrm{~Hz})$ for which the excitatory input in one cycle has little effect on the next cycle; and a high frequency $(380 \mathrm{~Hz})$, for which temporal summation of excitatory inputs activates subthreshold negative feedback thereby affecting subsequent spike generation. For comparing the influence of inhibition features we choose to define our control conditions so that each model has an approximately identical firing rate. We describe first the results for low frequency input with control conditions: for each model, set an EPSG value so that 6 simultaneous inputs elicit a spike and set the $b_{e}$ value to have a firing rate of about $50 \mathrm{~Hz}$.

The dependence of firing probability on the phase of inhibition shows a common feature nearly throughout our parameter variation set: firing probability is reduced nearly to zero when inhibition is timed close to excitation. This reduction effect can be very localized in time but for some other phases firing probability shows prominent increases. For time focused inhibition $\left(b_{i}>6\right.$, say) the firing probability increases by a factor of 1.5-2 for inhibition that just precedes excitation by $30-40 \%$ i.e., $\approx 2.5 \mathrm{~ms}$ (Figure 10A). This enhancement is more sharply tuned in phase for $\mathrm{S}$ and $\mathrm{C}$ than for $\mathrm{D}$. The weaker phase dependence for $\mathrm{D}$ is consistent with the slower and monotonic decay of an IPSP in the case of D. The IPSP decay for S and C exhibits resonant like behavior with an overshoot and therefore a greater phase preference (see Figure S5). The phase ranges where the firing ratio is near one correspond to inhibition that arrives just after a spike in the previous cycle and too far ahead of the next excitatory event to have any effect on firing probability. Finally, if inhibition is not focused in time but rather smeared (low value of $b_{i}$ ) the firing probability is close to or less than the control value and without phase preference.

The time course of inhibitory transients significantly affects the PIF mechanism. If $g_{\text {inh }}(t)$ is too fast or too slow, relative to the intrinsic timescales of $V$ and the subthreshold negative feedback, PIF is precluded as shown in Dodla et al. (2006) for the $I_{\mathrm{KLT}}$ mechanism. For our models and with repetitive excitatory input, as $\tau_{i}$ decreases and becomes small enough, the briefer $g_{\text {inh }}$ leads to a sharpening of the phase preference (Figure 10B) and stronger enhancement in firing probability. In contrast, as $\tau_{i}$ increases the phase preference tuning broadens and enhancement reduces giving way to a broadening of the late phase range in which firing probability is reduced below the control level $(\sim 50 \mathrm{~Hz})$. This reduction is especially true for the $\mathrm{D}$ model; for $\tau_{i}=0.9 \mathrm{~ms}$ the firing probability is below control values for approximately $80 \%$ of the cycle. The slower decay of an IPSP for D than for S and C (again, Figure S5) is exaggerated by larger $\tau_{i}$.

Stronger inhibition that is focused and brief can enhance the PIF effect (Figure 10C). The firing probability increases and the phase preference sharpens for phases just before the excitation event (approximately $1.7 \mathrm{~ms}$ in advance). The behaviors resemble those for increasing the vector strength of inhibition (cf, Figure 10A).
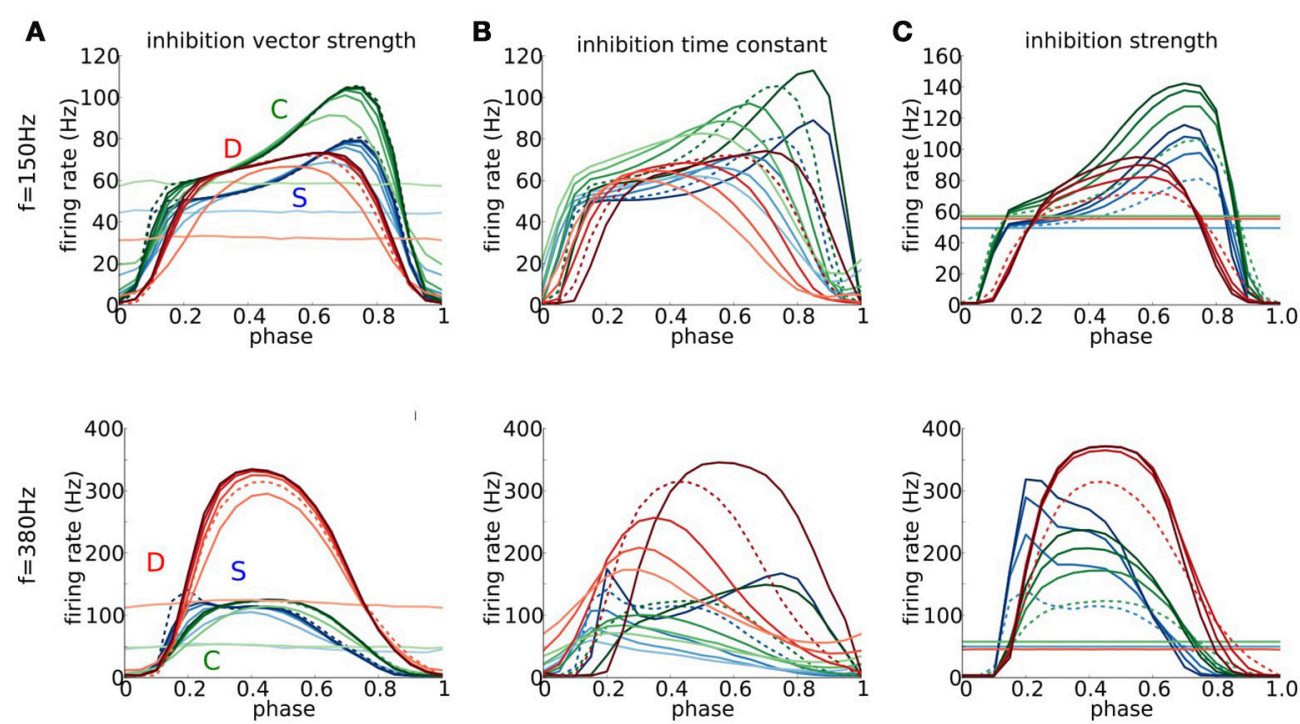

FIGURE 10 | The role of timed inhibition in signal detection at low and high frequencies. (A-C) Output firing rate for periodic trains of combined excitatory and inhibitory multi-synaptic inputs for the $\mathrm{D}(\mathrm{red}), \mathrm{S}$ (blue), and $\mathrm{C}$ (green) models, as a function of phase of inhibition with respect to excitation for $f=150 \mathrm{~Hz}$ (top) and $f=380 \mathrm{~Hz}$ (bottom). Parameters for the excitatory input are fixed so that the output firing rate in the absence of inhibition is about $50 \mathrm{~Hz}$ (for $f=150 \mathrm{~Hz}, b_{e}=15$ (S) $b_{e}=4(\mathrm{D}), b_{e}=12(\mathrm{C})$, and for $f=380 \mathrm{~Hz}, b_{e}=15$ (S) $b_{e}=3(\mathrm{D}), b_{e}=4$ (C)). Maximal conductance for mEPSGs $g_{\max , e}$ at $f=150 \mathrm{~Hz}$ is chosen so that six coincident inputs exceed threshold and at $f=380 \mathrm{~Hz}$ so that four coincident inputs exceed threshold. Parameters modeling the inhibitory multi-synaptic input are varied. (A) We vary the temporal coherence of the inhibitory inputs, ranging from $b_{i}=0$ (light colors) to $b_{i}=10$ (dark colors) in intervals of 2 ; here $\tau_{j}=0.3 \mathrm{~ms}$ and $g_{\max , i}=2.5 \mathrm{nS}$. (B) We vary the time scale of inhibitory inputs $\tau_{i}$, ranging from $\tau_{j}=0.1 \mathrm{~ms}$ (fast, dark colors) to $\tau_{j}=0.9 \mathrm{~ms}$ (slow, light colors) in intervals of $0.2 \mathrm{~ms}$; here $b_{i}=b_{e}$ and $g_{\max , i}$ is scaled so that the total area under the $\alpha$-function modeling IPSGs remains constant and takes the same value as for $\tau_{j}=0.3 \mathrm{~ms}$. (C) We vary the inhibiton strength $g_{\max , i}$, ranging from $g_{\max , i}=0 \mathrm{nS}$ (light colors) to $g_{\max , i}=10 \mathrm{nS}$ (dark colors) in intervals of $2.5 \mathrm{nS}$; here $b_{i}=b_{e}$ and $\tau_{i}=0.3 \mathrm{~ms}$ are fixed. Dashed curves identify the curves in (A-C) that share the same parameters for inhibition. 
Notice that the $\mathrm{C}$ model exhibits in Figure 10 (top) more enhancement of firing probability by brief inhibition overall than $\mathrm{D}$ or $\mathrm{S}$, in contrast to our comparisons of other response features (say, Figures 2, 4, 7) where $\mathrm{C}$ appeared intermediate in degree. The difference is reflecting the fact that the $\mathrm{C}$ model includes both mechanisms of subthreshold negative feedback. Hence, transient inhibition is capable of inducing extra hyperexcitability.

The behaviors for high frequency $(380 \mathrm{~Hz})$ excitatory input differ somewhat, but not surprisingly, from those for low frequency input (Figure 10, lower panels; see caption for control conditions). Overall, the $\mathrm{D}$ model shows the greatest enhancement in firing probability with transient inhibition. This effect is understandable since hyperpolarization from transient inhibition must overcome for $\mathrm{S}$ and $\mathrm{C}$ two factors: the temporal summated excitatory conductance between input events (the only hurdle for D) and the accumulated $g_{\text {KLT }}$. The D model shows less phase preference for PIF. For S and C the preferred phases are shifted earlier. These shifts correspond to $1.6-2.0 \mathrm{~ms}$ before the next input event (comparable in absolute time to that for the low frequency input case), a time scale comparable to $\tau_{w}$ and $\tau_{h}$. Interestingly, here for high frequency input the $\mathrm{C}$ model shows a much weaker PIF effect than S, although having a slightly larger effect than $S$ for low frequency input.

While timed inhibition can boost firing probability for peri-threshold random synaptic events it can also enhance coincidence detection and precision. We assessed in Figure 7 coincidence detection quality by showing the dependence on $b$ (the degree of temporal coherence in the input) for repetitive excitatory input; a horizontal slice in the heat map shows, for a given frequency, the increase of firing probability vs. $b$ (Figure 11A, dashed curves for $250 \mathrm{~Hz}$ input and low strength, as in Figure 7A). The effect of brief inhibition on this sensitivity to coincidence depends on the phase of inhibition (indicated with color intensity). In this view we recover the impression that the $\mathrm{C}$ model responds to coincidence with sensitivity intermediate to that of $\mathrm{S}$ and $\mathrm{D}$; the firing probability and the "dynamic range" (interval of $b$-values for gradation of firing probability) are intermediate. The $\mathrm{S}$ model, with its subtractive mechanism, has the largest dynamic range while the $\mathrm{D}$ model is coincidencesensitive over only a narrow range before saturation with firing probability exceeding 0.9 for $b>5$ or so. For each model we see that even when inhibition occurs close to excitation (dark curves) the low firing probability can be increased with coincidence. These results suggest that timed inhibition may modulate the rate encoding and dynamic range for coincidence detection of phasic cells, creating windows over different $b$ ranges.

The temporal precision (vector strength) of the models' responses increases with temporal coherence (Figure 11B). Comparison between the models shows $\mathrm{C}$ as intermediate and $\mathrm{D}$ with least precision. For timed inhibition, a high VS can always be achieved for strongly coincident inputs. In contrast, the VS is limited in the presence of noise even for very high $b$-values (compare Figure 9B and Figure 11B).

\section{DISCUSSION}

Phasic neurons do not respond repetitively to steady inputs. The primary mechanism for phasicness is a dynamic negative feedback mechanism that activates for subthreshold values of voltage, preventing the generation of a spike. Here we explored two mechanisms of different nature: a potassium current (outward current) that activates subthreshold and opposes depolarization (subtractive mechanism) and a transient sodium current (inward current) that inactivates subtreshold and can overwhelm activation gating of the regenerative current (divisive mechanism). We considered two reduced models that isolated these mechanisms, one that has only subtractive mechanism (S model), and one that has only divisive mechanism (D model); and a third model that combined both ( $\mathrm{C}$ model), and compared their performance under different stimuli.
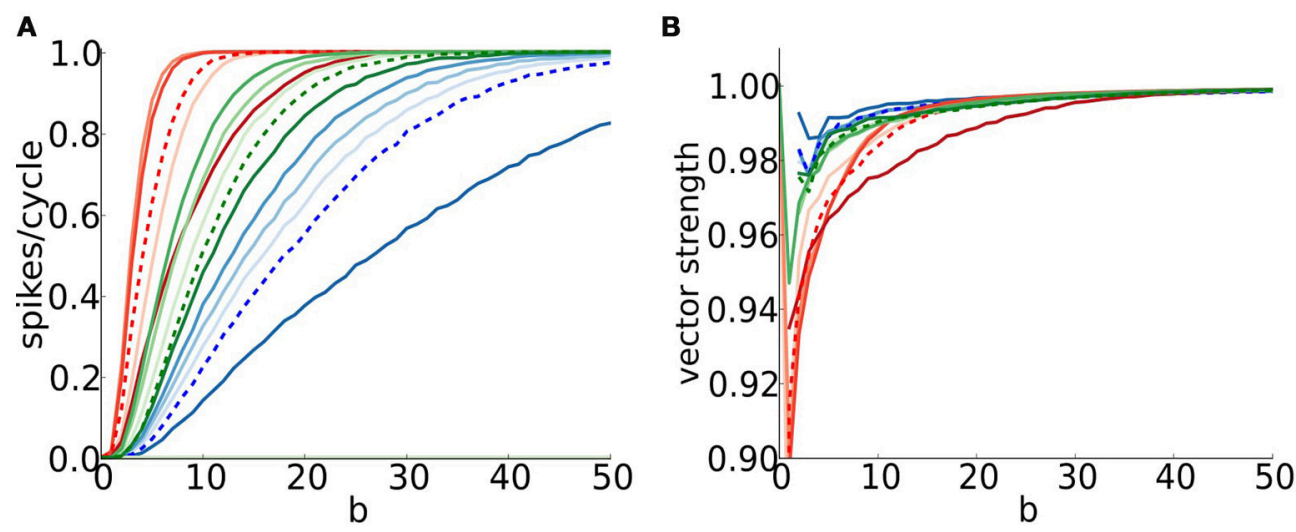

FIGURE 11 | The timing of inhibition can affect coincidence detection and vector strength. (A) Firing probability (spikes/cycle) and (B) vector strength for S (blue), D (red), and $\mathrm{C}$ (green) models, input frequency $f=250 \mathrm{~Hz}$ and low input strength (six coincident inputs exceed threshold for a spike) as a function of input temporal coherence $b$ ( $x$-axis) and phase difference between inhibition and excitation indicated with color intensity of curves $(0.2=$ light and $0.8=$ dark in steps of 0.2). Recall that for values of phase difference close to 0 , inhibition just follows excitation, while for values close to 1 , inhibition just precedes excitation. We include the results for no inhibition (dashed curves) for comparison. 
We found that each mechanism, divisive and subtractive, alone can support type III excitability. We do not advocate choosing one, say subtractive, as the default without consideration of the other. By extension, one should not assume that if an $I_{\mathrm{KLT}}$ blocker is applied (or $g_{\mathrm{KLT}}$ is replaced with a frozen $g_{\mathrm{KLT}}$, by dynamic clamp) and type III persists that $I_{\mathrm{KLT}}$ is not playing a role in Type III. The effect could have unmasked a D-mechanism, meriting further study.

Firing in type III excitability requires that the voltage outraces the dynamic negative feedback before this feedback can activate significantly and disallow a spike. This speed requirement on voltage rise means that these neurons and neuron models can act as input slope detectors. We found that our model with a subtractive mechanism requires a faster input rise than models without it (see ramps and sinusoids in Figure S1). Indeed, the (subtractive) low-threshold activated potassium current directly competes with a rising voltage by providing high conductance, that shunts EPSCs as well as noise fluctuations. The divisive mechanism of $\mathrm{Na}^{+}$inactivation does not interfere with the rising voltage but rather hinders the regenerative power of the sodium current. For this reason, we assert that cells equipped with $I_{\mathrm{KLT}}$ are better-suited for temporal processing like phase-locking and coincidence detection and with extraordinary temporal precision than neurons with only subthreshold $\mathrm{Na}^{+}$inactivation (see Figures 7, 8).

However, neurons without sodium inactivation, need to strongly reduce the maximum $\mathrm{Na}^{+}$conductance in order to preserve type III excitability, thus compromising excitability and ultimately signal detection (see Figure 3). Neurons equipped with both mechanisms, show the best combination of coincidence detection and signal detection phasic properties in addition to being the more robust to variations in channel densities. Indeed, the subtractive mechanism, when accompanied by a sodium current that inactivates (even if not subthreshold), increases its excitability while maintaining phasic properties. The divisive mechanism, if combined with a subthreshold activated potassium $I_{\mathrm{KLT}}$, becomes more selective to coincident inputs.

We provided new clues to assist in hypothesizing about mechanism(s) that may underlie phasic behavior in a given system. For instance, one can expect very differently shaped input-output relations (Figure 4) depending on whether S or $\mathrm{D}$ is dominant in determining Type III behavior; the relations for $\mathrm{D}$ are rather symmetric and not so for $\mathrm{S}$ and, for $\mathrm{S}$, the asymmetry interestingly switches if firing probability is plotted vs. input strength or plotted vs. $\langle V\rangle$. Moreover, the effect of increased inhibition onto these curves shows different trends: decreasing the firing rate monotonically in the $S$ model while affecting firing rate non-monotonically in the D model (Figure 5).

Given a particular system, one's approach for investigating and rationalizing the mechanistic basis should consider the context, the firing regimes. For instance, one could imagine a neuron with phasic spike capability at hyperpolarized levels, with an $I_{\mathrm{Na}}$ that differs from the $I_{\mathrm{Na}}$ that supports spike capability at depolarized levels. In this case, we might predict in favor of a D mechanism and against $S$ as the default. Indeed, $S$ would involve a substantial resting conductance that would require exceptionally strong excitatory input to elicit spikes with depolarization.

\subsection{Type III Excitability, Differentiators par excellence}

Neurons that mainly fire due to rapid depolarization to threshold caused by time coincident inputs (like the models considered herein) are often called differentiatiors (Izhikevich, 2007; Prescott et al., 2008a; Lundstrom et al., 2009; Ratte et al., 2014). Although some neurons may display features of integrators and differentiators depending on the temporal pattern of the inputs (Gutkin et al., 2003; Rudolph and Destexhe, 2003), here we have focused on pure neural differentiatiors which, because of their intrinsic properties, are incapable of responding to temporally integrated, slowly varying input. This property is strongly linked to the concept of type III excitability. That is, the system approximately tracks a slowly varing input as a pseudo steady state -there is no bifurcation to oscillatory behavior with the input treated as a parameter. Input variability is the essential cause of spiking (Lundstrom et al., 2009, 2008). This excitability type complements the classical type I and type II excitability classes, where a stable fixed point destabilizes through a SNIC or Hopf bifurcation, respectively (Rinzel and Ermentrout, 1998; Borisyuk and Rinzel, 2005; Izhikevich, 2007; Prescott et al., 2008a; Meng et al., 2012; Rinzel and Huguet, 2013).

Neurons with type II excitability (emergence of repetitive firing via a Hopf bifurcation) are typically classified as resonators since the emergence of repetitive firing is via a Hopf bifurcation and thereby associated with subthreshold damped oscillations (some type III models can also show damped oscillations and thus resonator properties Prescott et al., 2008a,b; Mikiel-Hunter et al., 2016). However, such a system may display differentiatorlike features when operating near but outside its repetitive firing regime (Prescott et al., 2008a; Ratte et al., 2014). The sensitivity to timing of multiple inputs, coincidence detection, is naturally inherited from the time windows of opportunity provided by subthreshold resonance. Some differentiator properties that we studied here are found exclusively in type III excitable systems. For instance, the fact that, in the presence of noise, pure differentiators have highly non-monotonic input-output curves and its firing rate is sensitive to variance and insensitive to mean input in the noise free case (see Figure 4). Moreover, in the present study, we added an extra ingredient: when the nonmonotonic input-output curves are plotted as a function of the mean voltage (see Figures $4 \mathbf{B}, 5 \mathrm{~B}$ ), the firing rate decay occurs for subthreshold values of voltage (below $\sim-40 \mathrm{mV}$; when $m$ is half activated), irrespective of the negative feedback mechanism considered. This property does not occur for type II neurons, for which the firing rate increases with mean input and the decay in firing rate may occur but at a much higher activated state.

\subsection{Two Mechanisms are Better than One to Provide Robustness to Neuron Models}

Previous studies on specialized coincidence detectors in the auditory brainstem (Manis and Marx, 1991; Rathouz and Trussell, 1998; Svirskis et al., 2002) have highlighted the relevance of $I_{\mathrm{KLT}}$ in conferring phasic properties. Our early finding that $\mathrm{Na}^{+}$current in MSO neurons inactivated at lowvoltages suggested that this feature contributes to enhancing 
coincidence detection (Svirskis et al., 2004). Here, we underscore that $\mathrm{Na}^{+}$inactivation plays a strong role in guaranteering the functional properties of the neuron model in terms of both spike preservation and phasicness. Thus, if $\mathrm{Na}^{+}$inactivation is disabled, as in the $\mathrm{S}$ model, sodium conductance must be tightly constrained in order to preserve the spike and avoid the "lockup" state (see Figure 3). We tried but were not able to counteract "lockup" and retain phasic behavior with an additional potassium current that activated at high voltage (see Appendix B in Supplementary Material). With the divisive mechanism alone, spiking is preserved if $h_{\infty}$ is left-shifted to lower values of voltage $-I_{\mathrm{Na}}$ must be strongly inactivated at rest - to retain type III excitability and avoid repetitive firing in response to steady current. However, having both types of negative feedback mechanisms in the neuron model ( $\mathrm{C}$ model) provides a larger parameter space for conductances over which the system preserves phasicness, without compromising the spike or the excitability of the system. Thus, phasic models equipped with two mechanisms of different nature are more robust to changes in channel density (see Figure 3A).

In our analysis we considered extreme cases for our models. Indeed, it is not necessary that a $\mathrm{C}$ model, being phasic and performing properly, remain phasic when the subtractive mechanism is disabled. For example, the original RM03 model is phasic but behaves tonically when $I_{\mathrm{KLT}}$ conductance is frozen (Meng et al., 2012). Actually, there is a range of admissible positionings of $\mathrm{Na}^{+}$inactivation for a $\mathrm{C}$ model that guarantee similar phasic features as our $\mathrm{C}$ model, even when the $\mathrm{D}$ model (frozen $w$ ) is not phasic. However, as $h_{\infty}$ is right-shifted, the phasic region for the $\mathrm{C}$ model is reduced and when the shift is too dramatric, it resembles our $\mathrm{S}$ model. In this sense, our $\mathrm{S}$ model is a limiting case of the $\mathrm{C}$ model. Thus, our parameter choice for models allow us to, on one hand, clearly label them as $\mathrm{S}$ or $\mathrm{C}$ and on the other hand, keep the parameter values as similar as possible for a fair comparison. See more details in Figure S6.

We have considered the idealized situation of a point neuron model, without regard for potential effects of differential spatial distributions of ion current mechanisms for phasic behavior. MSO neurons provide a case for such considerations. In MSO neurons somatically recorded spikes are typically quite weak in vitro (Scott et al., 2007) and distinguishing what might be spikes or synaptic transients is challenging from in vivo extracellular recordings. Spikes that are generated in the axon initial segment are presumably shunted by the considerable conductance of $I_{\mathrm{KLT}}$ and $I_{h}$ in the soma-dendritic membrane (Khurana et al., 2011), even though $\mathrm{Na}^{+}$channels (inactivated near rest) are present in the soma (Scott et al., 2010). A thorough modeling study of how MSO phasic firing may depend on both $g_{\mathrm{KLT}}$ and $\mathrm{Na}^{+}$inactivation could include the spatial distribution of the various $V$-gated currents (Ko et al., 2016); the influence of spatial distribution should likewise be considered in other phasic firing neurons. MSO neurons are superb at computing time differences. In gerbil, they receive fast excitation and fast, somatargeted, inhibition (Myoga et al., 2014). Perhaps the sodium current while inactivated at rest may be recruited (de-inactivated) by transient inhibition to respond selectively to well-timed inhibitory-then-excitatory event pairings as in post-inhibitory facilitation (Dodla et al., 2006).

Our findings can be extended to analyze the role of negative feedback processes in a wide range of biological rhythms. Indeed, the presence of two mechanisms of different nature opposing to depolarization is not exclusive of phasic systems. Thus, the original Hodgkin-Huxley model has also $\mathrm{Na}^{+}$inactivation and $\mathrm{K}^{+}$activation, which in this case, act mainly superthreshold. To counteract a strong non-inactivating inward current with a strong outward current constrains the allowable channel density. However, controlling the effect of autocatalysis by a divisive mechanism, makes the system less sensitive and more flexible to changes in channel density (Sengül et al., 2014). Spiking, crucial for neuronal communication and computation, needs to be preserved with respect to variations in channel density. A similar statement about "two better than one" can be applied to a network of excitatory and inhibitory neurons, say as in a firing rate framework like the Wilson-Cowan equations. In the classical formulation, inhibition acts as a subtractive mechanism to counter recurrent excitation (equivalent to sodium activation). Dynamic synaptic depression on the excitatoryexcitatory interaction behaves divisively like sodium inactivation by directly controlling and reducing autocatalysis. Having both mechanisms enables a network model to behave phasically (Tabak et al., 2006, 2011).

In further regard to robustness and parameter choices in our study, we acknowledge some compromises. Since we needed to choose a low value of $\bar{g}_{\mathrm{Na}}$ for the S model, the excitability for this model was reduced. Thus, in order to keep a fair comparison with the other models, we also reduced $\bar{g}_{\mathrm{Na}}$ for D and C models with respect to the original RM03 model, although this was not necessary since D and $\mathrm{C}$ still are type III for a larger range of $\bar{g}_{\mathrm{Na}}$ (see Figure $3 \mathbf{A}$ ). Lowering $\mathrm{Na}^{+}$conductance reduces, of course, the excitability properties of the system, and leads to lower amplitude spikes. Lowering $\bar{g}_{\mathrm{Na}}$ was also suggested in Lundstrom et al. (2008) as a mechanism to turn an integrator into a differentiator.

\subsection{The Role of Inhibition in Restoring Excitability and Shaping Coincidence Detection Properties}

Transient inhibition can reduce or, surprisingly, enhance firing probability for peri-threshold random synaptic input events, depending on the timing and precision of the inhibition (Dodla et al., 2006). This property of post-inhibitory facilitation (Dodla et al., 2006) is a transient analog for post-inhibitory rebound after prolonged hyperpolarization. It can be expected in any system with a dynamic excitability-suppressing factor that is partially activated at rest and reducible by transient hyperpolarization or in spontaneously firing conditions for randomly arriving excitatory and inhibitory events (Dodla and Rinzel, 2006). As a model, we considered the response sensitivity for periodically delivered, compound excitatory input events (say multi-synaptic inputs) that are near to threshold but by chance are sometimes subthreshold/superthreshold and more or less time dispersed. Our models (S and C) with $I_{\mathrm{KLT}}$ express the behavior. Inhibition 
reduces the excitability-suppressing $I_{\text {KLT }}$ current and although simultaneously positioning membrane potential further from threshold it opens a time window of enhanced excitability if inhibitory conductance decays fast enough (see Figures 5, 10). However, when excitability suppresion is primarily due to $\mathrm{Na}^{+}$ inactivation as in the $\mathrm{D}$ model, hyperpolarization from a transient inhibitory input can remove some $\mathrm{Na}^{+}$inactivation and thereby effectively lower the spike threshold in spite of hyperpolarization. A normally subthreshold excitatory input may then trigger a spike even if inhibition has not completely decayed away (see Figures 5, 10). The $\mathrm{C}$ model should behave more as the $\mathrm{S}$ model or the $\mathrm{D}$ model depending on the contribution of each mechanism. Thus, the PIF phenomenon for the D model is less sensitive to the arrival time of the inhibitory inputs, compared to $\mathrm{S}$ and $\mathrm{C}$, and this is especially noticeable at high frequencies.

While timed inhibition can facilitate signal detection, it can also enhance coincidence detection and precision. Several studies have shown that timed inhibition close to excitation, either preceeding or following excitation, can improve temporal precision of neurons (not only neurons with type III) by narrowing the window for coincidence detection, sometimes at the price of reducing the firing rate (Brand et al., 2002; Grothe, 2003; Ingham and McAlpine, 2005; Kuenzel et al., 2011). Indeed, we observed that timed inhibition close to excitation can deselect some spikes that do not correspond to coincident inputs and improve precision, especially for the $\mathrm{S}$ and $\mathrm{C}$ models, but also modulate the rate encoding and dynamic range of temporal coherence for coincidence detection (interval of $b$-values for gradation of firing probability), creating windows over different $b$ ranges (see Figure 11).

\subsection{Generality of the Model and the Role of Other Currents}

Many of our results are demonstrated for a specific conductancebased model (adapted from Rothman and Manis, 2003). However, the geometrical analysis and underlying mathematical structure of our reduced versions of the model suggest that qualitative aspects of phasicness will be found in a more general class of phasic models. Take for instance, Clay's model for a healthy squid giant axon (Clay et al., 2008), that was obtained from the original Hodgkin-Huxley $(\mathrm{HH})$ model by steepening and left-shifting the activation of $I_{\mathrm{K}}$. The modified model can be seen as our $\mathrm{C}$ model, since $\mathrm{HH}$ has sodium inactivation. Another possibility is to turn the standard $\mathrm{HH}$ model into a type III excitable model by lowering the conductance of $I_{\mathrm{Na}}$ from 120 to $83 \mathrm{mS} / \mathrm{cm} 2$ (Lundstrom et al., 2009). In this case, the model can be seen as our D model but with a potassium current that activates superthreshold or close to threshold.

Indeed, we may have kept the high threshold potassium current $I_{\mathrm{KHT}}$ (present in the original RM03 model) in the $\mathrm{D}$ model. The role of this current is to contribute to the repolarization of the membrane potential once the spike has occurred - recall that in the D model the repolarization is only due to the leak current - but it has no effect on the spike generation, since this current is not active at subthreshold values of voltage. For this reason, the results described for the D model will also follow if $I_{\mathrm{KHT}}$ is added, since they mainly involve the subthreshold dynamics. Some differences will be observed if the activation of $I_{\mathrm{KHT}}$ is shifted to lower values, closer to threshold (around $-40 \mathrm{mV}$ for instance). In this case, $I_{\mathrm{KHT}}$ will behave as an $I_{\mathrm{KLT}}$ current, so the model will become a $\mathrm{C}$ model, with proper scaling of conductances.

Similarly, the $I_{\mathrm{KHT}}$ current can also be included in the $\mathrm{C}$ and $\mathrm{S}$ models. For the $\mathrm{C}$ model, the inclusion of $I_{\mathrm{KHT}}$ does not change the behavior of the model significantly (Meng et al., 2012). The $S$ model is different since the inclusion of $I_{\mathrm{KHT}}$ might compensate the persistent $I_{\mathrm{Na}}$, and allow for a larger value of $\bar{g}_{\mathrm{Na}}$. Unfortunately, when $I_{\mathrm{KHT}}$ is incorporated in the S model, the system then switches to type II excitability (see Appendix B in Supplementary Material for a detailed discussion on this topic).

In many phasic models one can find a hyperpolarizationactivated cation current $I_{h}$ (Rothman and Manis, 2003; Khurana et al., 2011). The current $I_{h}$ is mostly activated below $V_{\text {rest }}$ with an activation time constant of $200-300 \mathrm{~ms}$ and deactivation by membrane depolarization on the order of tens of milliseconds. In our reduced models we have frozen $g_{h}$ to its resting value, which is small. Allowing dynamic $I_{h}$ may have an influence only on those results that involve dynamics below $V_{\text {rest }}$. This will constraint the range of voltages for negative currents, because $I_{h}$ prevents excessive hyperpolarization, in the same way as $I_{\mathrm{KLT}}$ prevents excessive depolarization. Moreover, when $I_{h}$ is activated the input resistance is lower at values of voltage below $V_{\text {rest }}$, thus the responsiveness to noise is smaller. The effects of a dynamic $I_{h}$ are more noticeable in the $\mathrm{C}$ and $\mathrm{S}$ model than in the $\mathrm{D}$ model. Indeed, $g_{\mathrm{KLT}}$ is frozen at the resting value for the D model, therefore, when voltage hyperpolarizes, dynamic $I_{\mathrm{KLT}}$ deactivates for $\mathrm{S}$ and $\mathrm{C}$, while for the D model it remains partially activated causing a similar effect on input resistance as if $I_{h}$ were present: input resistance is smaller and there is less probability to fire due to noise effects. Thus, the presence of $I_{h}$ (with dynamic rather than frozen-at-rest conductance) will have little effect on the essential results regarding coincidence detection properties. Even in the presence of inhibition, when $I_{h}$ may reduce the IPSP size, the results will remain valid but for a stronger inhibitory input.

\section{GENERAL CONCLUSION}

Subthreshold subtractive and divisive mechanisms each may contribute to, and together synergize to enhance, a cell's phasic properties. If they work alone, cell's performance may be compromised. Thus, the subtractive mechanism confers extraordinary coincidence detection properties to the cell, but, if alone (with non-inactivating sodium current), is operative within a restricted parameter range. The divisive mechanism guarantees robustness of phasic properties, without reducing a cell's excitability, although with somewhat less precision.

\section{AUTHOR CONTRIBUTIONS}

Conceived the theoretical framework: GH, XM, and JR. Designed the models and performed numerical simulations 
and mathematical analysis: $\mathrm{GH}$ and XM. Wrote the paper: $\mathrm{GH}, \mathrm{XM}$, and JR. Edited the manuscript: $\mathrm{GH}, \mathrm{XM}$, and JR.

\section{ACKNOWLEDGMENTS}

The research for this study was supported, in part, by the National Institutes of Health grant DC008543-01 (JR and XM), a Swartz Foundation fellowship, Ramon y Cajal fellowship 6-RYC-2014-

\section{REFERENCES}

Azouz, R., and Gray, C. M. (2000). Dynamic spike threshold reveals a mechanism for synaptic coincidence detection in cortical neurons in vivo. Proc. Natl. Acad. Sci. U.S.A. 97, 8110-8115. doi: 10.1073/pnas.130200797

Borisyuk, A., and Rinzel, J. (2005). "Understanding neuronal dynamics by geometrical dissection of minimal models," in Models and Methods in Neurophysics (Les Houches Summer School 2003), eds C. Chow, B. Gutkin, D. Hansel, C. Meunier, and J. Dalibard (Elsevier), 19-72.

Brand, A., Behrend, O., Marquardt, T., McAlpine, D., and Grothe, B. (2002). Precise inhibition is essential for microsecond interaural time difference coding. Nature 417, 543-547. doi: 10.1038/417543a

Carr, C. E., and Macleod, K. M. (2010). Microseconds matter. PLoS Biol. 8:e1000405. doi: 10.1371/journal.pbio.1000405

Clay, J. R., Paydarfar, D., and Forger, D. B. (2008). A simple modification of the Hodgkin and Huxley equations explains type 3 excitability in squid giant axons. J. R. Soc. Interface 5, 1421-1428. doi: 10.1098/rsif.2008.0166

Day, M. L., Doiron, B., and Rinzel, J. (2008). Subthreshold $\mathrm{K}^{+}$channel dynamics interact with stimulus spectrum to influence temporal coding in an auditory brain stem model. J. Neurophysiol. 99, 534-544. doi: 10.1152/jn.00326.2007

Dodla, R., and Rinzel, J. (2006). Enhanced neuronal response induced by fast inhibition. Phys. Rev. E. Stat. Nonlin. Soft Matter Phys. 73:010903. doi: 10.1103/PhysRevE.73.010903

Dodla, R., Svirskis, G., and Rinzel, J. (2006). Well-timed, brief inhibition can promote spiking: postinhibitory facilitation. J. Neurophysiol. 95, 2664-2677. doi: $10.1152 /$ jn.00752.2005

Ferragamo, M. J., and Oertel, D. (2002). Octopus cells of the mammalian ventral cochlear nucleus sense the rate of depolarization. J. Neurophysiol. 87, 22622270. doi: 10.1152/jn.00587.2001

Gai, Y., Doiron, B., Kotak, V., and Rinzel, J. (2009). Noise-gated encoding of slow inputs by auditory brain stem neurons with a low-threshold $\mathrm{K}^{+}$current. J. Neurophysiol. 102, 3447-3460. doi: 10.1152/jn.00538.2009

Gai, Y., Doiron, B., and Rinzel, J. (2010). Slope-based stochastic resonance: how noise enables phasic neurons to encode slow signals. PLoS Comput. Biol. 6:e1000825. doi: 10.1371/journal.pcbi.1000825

Goldberg, J. M., and Brown, P. B. (1969). Response of binaural neurons of dog superior olivary complex to dichotic tonal stimuli: some physiological mechanisms of sound localization. J. Neurophysiol. 32, 613-636.

Grothe, B. (2003). New roles for synaptic inhibition in sound localization. Nat. Rev. Neurosci. 4, 540-550. doi: 10.1038/nrn1136

Gutkin, B., Ermentrout, G. B., and Rudolph, M. (2003). Spike generating dynamics and the conditions for spike-time precision in cortical neurons. J. Comput. Neurosci. 15, 91-103. doi: 10.1023/A:1024426903582

Higgs, M. H., Slee, S. J., and Spain, W. J. (2006). Diversity of gain modulation by noise in neocortical neurons: regulation by the slow afterhyperpolarization conductance. J. Neurosci. 26, 8787-8799. doi: 10.1523/JNEUROSCI.1792-06.2006

Higham, D. J. (2001). An algorithmic introduction to numerical simulation of stochastic differential equations. SIAM Rev. 43, 525-546. doi: $10.1137 /$ S0036144500378302

Hodgkin, A. L. (1948). The local electric changes associated with repetitive action in a non-medullated axon. J. Physiol. (Lond.) 107, 165-181. doi: 10.1113/jphysiol.1948.sp004260
15866 and CUR-DIUE grant 2009SGR859 (GH). GH wants to acknowledge the use of UPC Applied Math cluster system for research computing.

\section{SUPPLEMENTARY MATERIAL}

The Supplementary Material for this article can be found online at: http://journal.frontiersin.org/article/10.3389/fncom. 2017.00003/full\#supplementary-material

Ingham, N. J., and McAlpine, D. (2005). GABAergic inhibition controls neural gain in inferior colliculus neurons sensitive to interaural time differences. J. Neurosci., 25, 6187-6198. doi: 10.1523/JNEUROSCI.014605.2005

Izhikevich, E. M. (2007). Dynamical Systems in Neuroscience: The Geometry of Excitability and Bursting. Cambridge, MA: Computational Neuroscience. MIT Press.

Jercog, P. E., Svirskis, G., Kotak, V. C., Sanes, D. H., and Rinzel, J. (2010). Asymmetric excitatory synaptic dynamics underlie interaural time difference processing in the auditory system. PLoS Biol. 8:e1000406. doi: 10.1371/journal.pbio.1000406

Joris, P. X., Carney, L. H., Smith, P. H., and Yin, T. C. (1994). Enhancement of neural synchronization in the anteroventral cochlear nucleus. I. Responses to tones at the characteristic frequency. J. Neurophysiol. 71, 1022-1036.

Joris, P. X., and Smith, P. H. (2008). The volley theory and the spherical cell puzzle. Neuroscience, 154, 65-76. doi: 10.1016/j.neuroscience.2008. 03.002

Khurana, S., Remme, M. W., Rinzel, J., and Golding, N. L. (2011). Dynamic interaction of Ih and IK-LVA during trains of synaptic potentials in principal neurons of the medial superior olive. J. Neurosci., 31, 8936-8947. doi: 10.1523/JNEUROSCI.1079-11.2011

Ko, K. W., Rasband, M. N., Meseguer, V., Kramer, R. H., and Golding, N. L. (2016). Serotonin modulates spike probability in the axon initial segment through HCN channels. Nat. Neurosci. 19, 826-834. doi: 10.1038/nn.4293

Kuenzel, T., Borst, J. G., and van der Heijden, M. (2011). Factors controlling the input-output relationship of spherical bushy cells in the gerbil cochlear nucleus. J. Neurosci. 31, 4260-4273. doi: 10.1523/JNEUROSCI.543310.2011

Lundstrom, B. N., Famulare, M., Sorensen, L. B., Spain, W. J., and Fairhall, A. L. (2009). Sensitivity of firing rate to input fluctuations depends on time scale separation between fast and slow variables in single neurons. J. Comput. Neurosci. 27, 277-290. doi: 10.1007/s10827-009-0142-x

Lundstrom, B. N., Hong, S., Higgs, M. H., and Fairhall, A. L. (2008). Two computational regimes of a single-compartment neuron separated by a planar boundary in conductance space. Neural Comput. 20, 1239-1260. doi: $10.1162 /$ neco.2007.05-07-536

Manis, P. B., and Marx, S. O. (1991). Outward currents in isolated ventral cochlear nucleus neurons. J. Neurosci. 11, 2865-2880.

Mathews, P. J., Jercog, P. E., Rinzel, J., Scott, L. L., and Golding, N. L. (2010). Control of submillisecond synaptic timing in binaural coincidence detectors by $\mathrm{K}_{\mathrm{v}} 1$ channels. Nat. Neurosci. 13, 601-609. doi: 10.1038/nn.2530

McGinley, M. J., and Oertel, D. (2006). Rate thresholds determine the precision of temporal integration in principal cells of the ventral cochlear nucleus. Hear. Res. 216-217, 52-63. doi: 10.1016/j.heares.2006.02.006

Meng, X., Huguet, G., and Rinzel, J. (2012). Type III excitability, slope sensitivity and coincidence detection. Discrete Contin. Dyn. Syst. 32, 2729-2757. doi: $10.3934 /$ dcds.2012.32.2729

Mikiel-Hunter, J., Kotak, V., and Rinzel, J. (2016). High-Frequency Resonance in the Gerbil Medial Superior Olive. PLoS Comput. Biol. 12:e1005166. doi: 10.1371/journal.pcbi.1005166

Myoga, M. H., Lehnert, S., Leibold, C., Felmy, F., and Grothe, B. (2014). Glycinergic inhibition tunes coincidence detection in the auditory brainstem. Nat. Commun. 5, 3790. doi: 10.1038/ncomms4790 
Oertel, D. (1983). Synaptic responses and electrical properties of cells in brain slices of the mouse anteroventral cochlear nucleus. J. Neurosci., 3, 2043-2053.

Oertel, D. (1999). The role of timing in the brain stem auditory nuclei of vertebrates. Annu. Rev. Physiol. 61, 497-519. doi: 10.1146/annurev.physiol. 61.1.497

Platkiewicz, J., and Brette, R. (2010). A threshold equation for action potential initiation. PLoS Comput. Biol. 6:e1000850. doi: 10.1371/journal.pcbi.1000850

Platkiewicz, J., and Brette, R. (2011). Impact of fast sodium channel inactivation on spike threshold dynamics and synaptic integration. PLoS Comput. Biol. 7:e1001129. doi: 10.1371/journal.pcbi.1001129

Prescott, S. A., and De Koninck, Y. (2002). Four cell types with distinctive membrane properties and morphologies in lamina I of the spinal dorsal horn of the adult rat. J. Physiol. (Lond.) 539, 817-836. doi: 10.1113/jphysiol.2001.013437

Prescott, S. A., De Koninck, Y., and Sejnowski, T. J. (2008a). Biophysical basis for three distinct dynamical mechanisms of action potential initiation. PLoS Comput. Biol. 4:e1000198. doi: 10.1371/journal.pcbi.1000198

Prescott, S. A., Ratté, S., De Koninck, Y., and Sejnowski, T. J. (2008b). Pyramidal neurons switch from integrators in vitro to resonators under in vivo-like conditions. J. Neurophysiol. 100, 3030-3042. doi: 10.1152/jn.90634.2008

Rathouz, M., and Trussell, L. (1998). Characterization of outward currents in neurons of the avian nucleus magnocellularis. J. Neurophysiol. 80, 2824-2835.

Ratte, S., Lankarany, M., Rho, Y. A., Patterson, A., and Prescott, S. A. (2014). Subthreshold membrane currents confer distinct tuning properties that enable neurons to encode the integral or derivative of their input. Front. Cell Neurosci. 8:452. doi: 10.3389/fncel.2014.00452

Remme, M. W., Donato, R., Mikiel-Hunter, J., Ballestero, J. A., Foster, S., Rinzel, J., et al. (2014). Subthreshold resonance properties contribute to the efficient coding of auditory spatial cues. Proc. Natl. Acad. Sci. U.S.A. 111, E2339-E2348. doi: $10.1073 /$ pnas. 1316216111

Reyes, A. D., Rubel, E. W., and Spain, W. J. (1994). Membrane properties underlying the firing of neurons in the avian cochlear nucleus. J. Neurosci. 14, 5352-5364.

Rinzel, J., and Ermentrout, G. (1998). "Analysis of neural excitability and oscillations," in Methods in Neuronal Modelling: From Synapses to Networks, 2nd Edn., eds C. Koch and I. Segev (Cambridge, MA: MIT Press), 251-291.

Rinzel, J., and Huguet, G. (2013). Nonlinear dynamics of neuronal excitability, oscillations, and coincidence detection. Comm. Pure Appl. Math. 66, 14641494. doi: 10.1002/cpa.21469

Rothman, J. S., and Manis, P. B. (2003). The roles potassium currents play in regulating the electrical activity of ventral cochlear nucleus neurons. J. Neurophysiol. 89, 3097-3113. doi: 10.1152/jn.00127.2002
Rudolph, M., and Destexhe, A. (2003). Tuning neocortical pyramidal neurons between integrators and coincidence detectors. J. Comput. Neurosci. 14, 239251. doi: $10.1023 / \mathrm{A}: 1023245625896$

Schnupp, J. W., and Carr, C. E. (2009). On hearing with more than one ear: lessons from evolution. Nat. Neurosci. 12, 692-697. doi: 10.1038/ nn. 2325

Scott, L. L., Hage, T. A., and Golding, N. L. (2007). Weak action potential backpropagation is associated with high-frequency axonal firing capability in principal neurons of the gerbil medial superior olive. J. Physiol. (Lond.) 583(Pt 2), 647-661. doi: 10.1113/jphysiol.2007.136366

Scott, L. L., Mathews, P. J., and Golding, N. L. (2010). Perisomatic voltagegated sodium channels actively maintain linear synaptic integration in principal neurons of the medial superior olive. J. Neurosci. 30, 2039-2050. doi: 10.1523/JNEUROSCI.2385-09.2010

Sengül, S., Clewley, R., Bertram, R., and Tabak, J. (2014). Determining the contributions of divisive and subtractive feedback in the HodgkinHuxley model. J. Comput. Neurosci. 37, 403-415. doi: 10.1007/s10827-0140511-y

Svirskis, G., Kotak, V., Sanes, D. H., and Rinzel, J. (2002). Enhancement of signalto-noise ratio and phase locking for small inputs by a low-threshold outward current in auditory neurons. J. Neurosci. 22, 11019-11025.

Svirskis, G., Kotak, V., Sanes, D. H., and Rinzel, J. (2004). Sodium along with low-threshold potassium currents enhance coincidence detection of subthreshold noisy signals in MSO neurons. J. Neurophysiol. 91, 2465-2473. doi: 10.1152/jn.00717.2003

Tabak, J., O’Donovan, M. J., and Rinzel, J. (2006). Differential control of active and silent phases in relaxation models of neuronal rhythms. J. Comput. Neurosci. 21, 307-328. doi: 10.1007/s10827-006-8862-7

Tabak, J., Rinzel, J., and Bertram, R. (2011). Quantifying the relative contributions of divisive and subtractive feedback to rhythm generation. PLoS Comput. Biol. 7:e1001124. doi: 10.1371/journal.pcbi.1001124

Conflict of Interest Statement: The authors declare that the research was conducted in the absence of any commercial or financial relationships that could be construed as a potential conflict of interest.

Copyright (C) 2017 Huguet, Meng and Rinzel. This is an open-access article distributed under the terms of the Creative Commons Attribution License (CC BY). The use, distribution or reproduction in other forums is permitted, provided the original author(s) or licensor are credited and that the original publication in this journal is cited, in accordance with accepted academic practice. No use, distribution or reproduction is permitted which does not comply with these terms. 